\title{
Modelos para gestão de riscos em cadeias de suprimentos: revisão, análise e diretrizes para futuras pesquisas
}

\author{
Models for risk management in supply chains: review, analysis, \\ and guidelines for research
}

\author{
Robson Nogueira Tomas ${ }^{1}$ \\ Rosane Lúcia Chicarelli Alcantara²
}

\begin{abstract}
Resumo: O objetivo deste artigo é apresentar uma revisão bibliográfica, que possa ser considerada completa e inédita na literatura científica nacional, tendo como foco modelos para gestão de riscos em cadeias de suprimentos. Para tanto, foram revisados 103 artigos científicos publicados entre 2000 e 2012. Inicialmente, buscou-se categorizar os tipos de riscos abordados nos artigos científicos segundo a descrição proposta por Christopher e Peck (2004), a saber: internos à empresa focal (operações, processo e controle); externos para a empresa focal, mas internos para a cadeia (fornecimento e demanda); e externos à cadeia (macroambiente). Buscou-se, também, identificar as etapas elementares na composição de um modelo para gestão de riscos em cadeias de suprimentos. Uma vez categorizados os tipos de riscos e identificadas as quatro etapas elementares, efetuou-se uma análise a partir de três perspectivas: i) as opções de modelos disponíveis na literatura para a prática de tal gestão, considerando-se os tipos de riscos e etapas que os compõem; ii) um modelo final a ser proposto; e iii) elementos que podem ser acrescentados ao modelo final. As principais contribuições deste trabalho dizem respeito a possibilitar melhor entendimento dos tipos de riscos que podem incidir sobre uma cadeia de suprimentos, discutir os modelos mais adequados para geri-los, além de oferecer diretrizes para futuras pesquisas na temática em questão.
\end{abstract}

Palavras-chave: Riscos. Modelos de gestão. Cadeias de suprimentos. Revisão de literatura.

\begin{abstract}
The aim of this paper is to present a literature review which can be considered thorough and unprecedented in the Brazilian scientific literature focusing on models for risk management in supply chains. To this end, 103 studies published between 2000 and 2012 were reviewed aiming to categorize the types of risks addressed in previous studies, according to the description proposed by Christopher and Peck (2004): internal to the focal company (operations, process, and control); external to the focal company but internal to the supply chain (supply and demand); and external to the chain (macro-environment). Subsequently, the elementary steps in the development of a model for risk management in supply chains were identified. After the types of risks were categorized and four elementary steps were identified, an analysis was conducted based on three perspectives: i) options of models for the practice of management, considering the types of risks and their steps ii) development of a final model to be proposed, and iii) elements that could be added to the final model. The main contributions of this study are related to a better understanding of the types of risks posed within a supply chain and the most appropriate models to manage them. In addition, it suggests guidelines for future research on this topic.
\end{abstract}

Keywords: Risks. Management models. Supply chain. Literature review.

\section{Introdução}

A gestão de riscos na cadeia de suprimentos (Supply Chain Risk Management - SCRM) envolve a identificação e o controle dos riscos internos e externos que podem afetar o desempenho de uma cadeia, por meio de uma abordagem coordenada entre os membros, de forma a prevenir ou mitigar as vulnerabilidades da cadeia como um todo
(CRANFIELD..., 2002; JÜTTNER; CHRISTOPHER; PECK, 2003; CHRISTOPHER, 2004). Cabe destacar que pesquisas envolvendo a gestão de riscos em cadeias de suprimentos, embora estejam em ascensão nos países desenvolvidos (PECK, 2006; CHRISTOPHER et al., 2011), ainda são pouco exploradas no Brasil (AGUIAR, 2010).

\footnotetext{
${ }^{1}$ Programa de Pós-graduação em Engenharia de Produção, Universidade Federal de São Carlos - UFSCar, São Carlos, SP, Brasil, e-mail: rn.tomas@uol.com.br

${ }^{2}$ Departamento de Engenharia de Produção, Universidade Federal de São Carlos - UFSCar, São Carlos, SP, Brasil, e-mail: rosane@ufscar.br
} 
De maneira geral, diversos autores (SIMCHI-LEVI; KAMINSKY; SIMCHI-LEVI, 2000; LAMBERT; POHLEN, 2001; MENTZER et al., 2001, por exemplo) têm destacado a gestão de uma cadeia de suprimentos como um meio importante para aprimorar os fluxos de produtos, serviços e informações, proporcionando custos reduzidos, entregas mais ágeis e confiáveis, a fim de agregar valor para o cliente final e criar vantagens comerciais competitivas a longo prazo. Entretanto, as cadeias de suprimentos são, por natureza, suscetíveis aos eventos de riscos (AGUIAR, 2010).

Há evidências de que fontes de riscos e incertezas permeiam todas as cadeias de suprimentos (RITCHIE; BRINDLEY, 2004). Pesquisas realizadas pelo Centro de Logística e Gestão da Cadeia de Suprimentos, da Universidade de Cranfield (CRANFIELD..., 2002) mostraram que, devido à complexidade e maior interdependência das empresas, ocorrida em todo o mundo nos últimos anos, o risco é um elemento constantemente presente nas cadeias de suprimentos, seja por meio de perturbações, de incertezas ou de interrupções. Dessa forma, pesquisas abordando a identificação e possível mitigação de perturbações (CHOPRA; SODHI, 2004; CHRISTOPHER; PECK, 2004), minimização de incertezas (JÜTTNER; CHRISTOPHER; PECK, 2003; PECK, 2005) ou diminuição de interrupções (SHEFFI; RICE, 2005; WAGNER; BODE, 2006) têm assumido um novo grau de relevância dentro das questões referentes à gestão de uma cadeia de suprimentos (PECK, 2005).

Neste contexto, Jüttner, Christopher e Peck (2003) apontam um caminho para a análise exploratória sobre este tema, ao afirmar que os riscos encontrados em uma cadeia formam o quadro de vulnerabilidades. Soma-se a isto Peck (2005), ao constatar que as vulnerabilidades da cadeia de suprimentos estão ligadas aos riscos, no sentido de que algo é passível de ser perdido ou danificado. Corroborando com esta perspectiva, Wagner e Bode (2008) e Ritchie e Brindley (2007) afirmam que, se os riscos interferirem no desempenho de uma cadeia, ela deverá ser submetida a um estudo objetivando a gestão e possível mitigação de tais elementos.

Notoriamente, todas estas pesquisas confirmam o crescente interesse, por pesquisadores de todo o mundo acerca das questões relativas aos riscos em cadeias de suprimentos. A presente revisão tem como objetivo geral contribuir para um melhor conhecimento no que se refere aos riscos e vulnerabilidades que podem incidir sobre uma cadeia de suprimentos, bem como apresentar os modelos mais adequados para geri-los. Além disso, no atual contexto de expansão da pesquisa brasileira, caracterizado por maiores investimentos em ciência, tecnologia e inovação, bem como pela recente inserção do Brasil entre os países que mais produzem pesquisas científicas no mundo, torna-se evidente a importância do desenvolvimento de estudos em campos de pesquisa pouco explorados, como é o caso do tema em questão. Tal fato justifica a presente revisão que, além de ser inédita na literatura científica nacional, pode contribuir para futuras investigações teóricas e empíricas, maiormente, no que se refere ao desenvolvimento de modelos para gestão de riscos aplicados à realidade de cadeias brasileiras.

O presente trabalho está organizado em seções. Nesta seção é apresentada a introdução e, na seção 2, a metodologia da pesquisa. Os tipos de riscos e as etapas para gestão são descritos na seção 3 . Na seção 4, apresenta-se a análise dos modelos e, na seção 5, são apresentadas as considerações finais, seguidas pelas referências bibliográficas, na seção 6 .

\section{Procedimentos metodológicos}

A presente pesquisa pode ser considerada exploratória, sendo que os resultados desejados foram alcançados por meio de uma revisão bibliográfica. Entende-se que a revisão bibliográfica é um passo essencial na estruturação de estudos em campos de pesquisa pouco explorados (EASTERBY-SMITH; THORPE; LOWE, 2002).

O processo de revisão foi composto por três estágios, conforme sugere Srivastava (2007), a saber: i) definição da unidade de análise; ii) escolha do contexto de pesquisa; e iii) delimitação da coleta de dados. Com relação à definição da unidade de análise, cabe destacar que se optou por coletar os dados a partir de artigos científicos publicados nos periódicos de maior relevância para a área de Gestão da Cadeia de Suprimentos, excluindo-se anais de congressos da unidade de análise. Com relação à escolha do contexto de pesquisa, optou-se por utilizar algumas das bases de dados disponíveis no portal de periódicos da CAPES, a saber, Ebsco, Scielo, Science Direct, Scopus, Scirus (Elsevier), além da base Emerald. No que diz respeito à delimitação da coleta de dados, focou-se a busca por documentos que continham as palavras-chave "supply chain risk management" no título e/ou resumo, para o caso de artigos redigidos em língua inglesa, e "gestão de riscos na cadeia de suprimentos", para a busca em periódicos nacionais (base Scielo). A busca na base Scielo foi realizada pela primeira vez em maio de 2012 e, pela segunda vez, em agosto do mesmo ano. Ressalta-se que nenhum artigo sobre o tema em questão foi encontrado nas duas buscas realizadas nesta base. A busca nas bases de periódicos da CAPES foi realizada em maio de 2012.

Outro critério utilizado como delimitação é referente ao fato de terem sido selecionados, apenas, os artigos publicados a partir do ano 2000. O referido ano foi selecionado como ponto de partida para a busca por se entender que as pesquisas mais relevantes para a Gestão de Riscos em Cadeias de Suprimentos ocorrem a partir deste ano, conforme indicam Ghadge, Dani e Kalawsky (2012). Corroboram com tal entendimento 
Ponomarov e Holcomb (2009, p. 130), ao mencionarem que a importância de SCRM para a tomada de decisões em uma cadeia de suprimentos aumentou significativamente desde o início dos anos 2000, devido, em grande parte " [...] a uma série de tendências como globalização, terceirização, a transição para operações enxutas e ágeis, ataques terroristas, e outras ameaças". Por isso, segundo os mesmos autores, as pesquisas mais relevantes sobre esta abordagem não antecedem o início dos anos 2000. Assim sendo, é este o motivo pelo qual a presente revisão ateve-se somente aos artigos publicados entre 2000 e 2012.

Com relação ao procedimento de seleção dos artigos, a busca por documentos que continham as palavras-chave "supply chain risk management" no título e/ou resumo resultou em um total inicial de 1.258 artigos (somando-se os artigos encontrados nas bases Ebsco, Science Direct, Scopus, Elsevier e Emerald). Em seguida, por meio de "filtros" disponibilizados dentro das bases de busca, efetuou-se uma separação dos artigos segundo suas áreas temáticas, sendo que foram selecionados para compor a presente revisão somente os artigos que tratavam de "riscos" no contexto da Gestão de Cadeias de Suprimentos, o que produziu uma amostra final de 170 artigos, somando-se todas as bases. Depois da eliminação de artigos repetidos (duplicatas), este número foi reduzido para 103 artigos (total final). A partir disso, iniciou-se uma ampla análise abordando a capacidade de abrangência dos riscos e os modelos mais adequados para serem aplicados em sua gestão, conforme será apresentado nas seções seguintes. A Figura 1 ilustra os passos utilizados para busca de artigos redigidos em língua inglesa.

\section{Tipos de riscos e etapas envolvendo um modelo de gestão}

\subsection{Descrições iniciais}

Antes mesmo do surgimento de pesquisas relacionadas à gestão da cadeia de suprimentos, a temática envolvendo gestão de riscos estava presente no foco de vários estudos relacionados ao ambiente organizacional (KNIGHT, 1965; BAIRD; THOMAS, 1985, por exemplo).

Entretanto, com a recente atenção dispensada por parte de pesquisadores de todo o mundo no que se refere à necessidade de coordenação entre fornecimento, produção e distribuição, a fim de que os produtos estejam disponíveis no tempo e lugar certo e na quantidade correta (MENTZER et al., 2001), a antiga priorização da cadeia de suprimentos, antes formada pela tríade "redução dos custos de produção, maior agregação de valor aos produtos, e, satisfação dos clientes", tem sido acrescentada, nos últimos anos, pela necessidade de gestão de suas vulnerabilidades e riscos (MARBLE; THUN, 2006, p. 08).

Zsidisin e Ritchie (2009) indicam que a teoria sobre gestão de riscos em cadeias de suprimentos (SCRM) é o resultado da interseção entre supply chain management (SCM) e risk management (RM), tal como mostra a Figura 2.

Sob esta perspectiva, cabe destacar que a gestão de riscos é uma abordagem recente no ambiente de cadeias de suprimentos (AGUIAR, 2010), pois parte significativa das pesquisas não data mais do que 12 anos, conforme é possível notar na Tabela 1, a qual apresenta as 103 publicações que compõem a presente revisão.

\subsection{Categorização dos tipos de riscos}

A presente revisão evidencia que são muitos os tipos de riscos que tem afetado as cadeias de suprimentos. Diversos autores têm proposto maneiras de se categorizar estes riscos, por exemplo: em relação aos fatores de causa e efeito (HALLIKAS; VIROLAINEN; TUOMINEN, 2002); pela ruptura potencial em relação à probabilidade versus consequência (SHEFFI; RICE, 2005); a partir de eventos gerados pelo ambiente interno e pelo ambiente

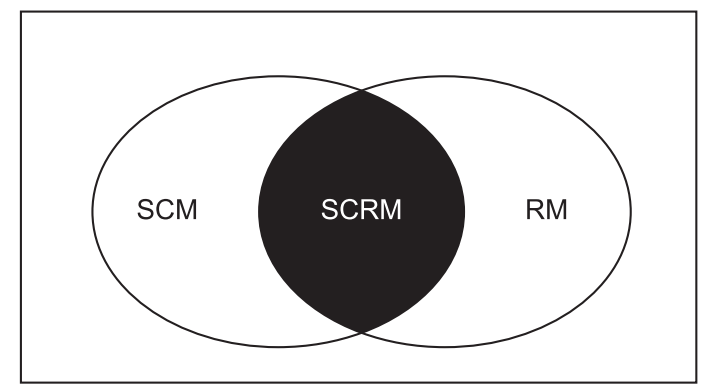

Figura 2. Área de interseção entre as teorias. Fonte: Adaptado de Aguiar (2010).

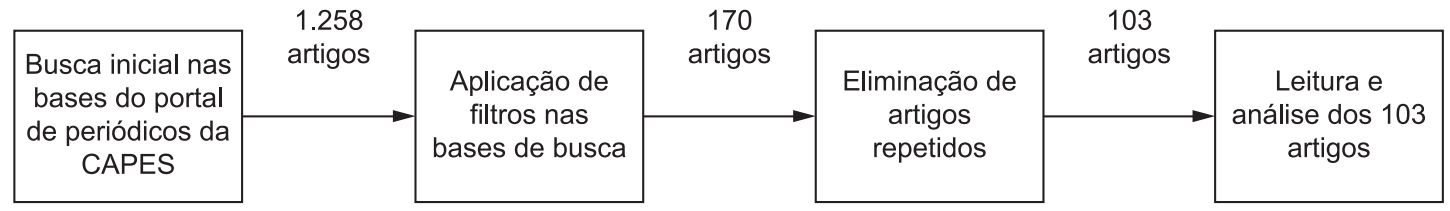

Figura 1. Procedimento utilizado para a busca de artigos. Fonte: Elaborado pelos autores. 
Tabela 1. Publicações que compõem a presente revisão.

\begin{tabular}{cl}
\hline Ano/Publicação & \multicolumn{1}{c}{ Autores } \\
\hline Até 2000* & $\begin{array}{l}\text { Halman e Keizer (1994), Fisher et al. (1997), Kleindorfer (1997), Mason-Jones e Towill (1998) } \\
\text { e Perrow (1999). }\end{array}$
\end{tabular}

De 2000 a $2004 \quad 1$ Christopher (2000), 2 Svensson (2000), 3 Zsidisin, Panelli e Upton (2000), 4 Johnson (2001), 5 Lee e Christopher (2001), 6 Sheffi (2001), 7 Hallikas, Virolainen e Tuominen (2002), 8 Lee (2002), 9 Svensson (2002a), 10 Svensson (2002b), 11 Svensson (2002c), 12 Svensson (2002d), 13 Harland, Brenchley e Walker (2003), 14 Hendricks e Singhal (2003), 15 Jüttner, Christopher e Peck (2003), 16 Meulbroek (2003), 17 Rice e Cainato (2003), 18 Svensson (2003a), 19 Svensson (2003b), 20 Zsidisin (2003), 21 Zsidisin e Ellram (2003), 22 Brindley (2004), 23 Ritchie e Brindley (2004), 24 Cavinato (2004), 25 Chopra e Sodhi (2004), 26 Christopher e Lee (2004), 27 Christopher e Peck (2004), 28 Finch (2004), 29 Giunipero e Eltantawy (2004), 30 Hallikas et al. (2004), 31 Hallikas e Virolainen (2004), 32 Norrman e Jansson (2004), 33 Norrman e Lindroth (2004), 34 Ritchie e Brindley (2004), 35 Speckman e Davis (2004), 36 Zsidisin et al. (2004), 37 Zsidisin (2004), 38 Zsidisin e Smith (2005).

De 2005 a 200939 Blackhurst et al. (2005), 40 Craighead et al. (2005), 41 Dimiturk (2005), 42 Hendricks e Singhal (2005), 43 Jüttner (2005), 44 Kleindorfer e Saad (2005), 45 Peck (2005), 46 Sheffi (2005), 47 Sheffi e Rice (2005), 48 Sodhi (2005), 49 Towill (2005), 50 Zeng, Berger e Gerstenfeld (2005), 51 Zsidisin, Melnyk e Ragatz (2005), 52 Cucchiella e Gastaldi (2006), 53 Gaudenzi e Borghesi (2006), 54 Faisal, Banwet e Shankar (2006), 55 Kouvelis, Chambers e Wang (2006), 56 Peck (2006), 57 Tang (2006a), 58 Tang (2006b), 59 Tomlin (2006), 60 Wagner e Bode (2006), 61 Wu, Blackhurst e Chidambaram (2006), 62 Faisal, Banwet e Shankar (2007), 63 Khan e Burnes (2007), 64 Levary (2007), 65 Perry (2007), 66 Ritchie e Brindley (2007), 67 Elkins, Kulkarni e Tew (2008), 68 Ellegaard (2008), 69 Faisal, Banwet e Shankar (2008), 70 Hallikas e Varis (2009), 71 Handfield (2008), 72 Handfield e McCormack (2008), 73 Jüttner e Ziengenbein (2009), 74 Khan, Christopher e Burnes (2008), 75 Manuj e Mentzer (2008a), 76 Manuj e Mentzer (2008b), 77 Matook, Lasch e Tamaschke (2008), 78 Melnyk, Rodrigues e Ragatz (2009), 79 Mullai (2009), 80 Smith (2009), 81 Viswanadham e Gaonkar (2008), 82 Tang e Tomlin (2008), 83 Wagner e Bode (2008), 84 Blos et al. (2009), 85 Laeequddin et al. (2009), 86 Ponomarov e Holcomb (2009), 87 Pujawan e Geraldin (2009), 88 Rao e Goldsby (2009), 89 Skipper e Hanna (2009).

Entre 2010 e 201290 Liu, Lin e Hayes (2010), 91 Jia e Rutherford (2010), 92 Olson e Dash Wu (2010), 93 Sanchez-Rodrigues, Potter e Naim (2010), 94 Christopher et al. (2011), 95 Elangovan et al. (2011), 96 Hans-Christian, Gallus e Thomas (2011), 97 Hittle e Leonard (2011), 98 Jüttner e Maklan (2011), 99 Lin e Zhou (2011), 100 Olson e Wu (2011), 101 Tummala e Shoenherr (2011), 102 Kern et al. (2012), 103 Shin et al. (2012).

*Alguns exemplos que não fazem parte da presente revisão.

externo das cadeias de suprimentos (TUMMALA; SCHOENHERR, 2011); entre outras. Entretanto, entende-se que tais abordagens são, na maioria das vezes, ou incompletas ou específicas demais, o que pode impossibilitar uma aplicação mais abrangente, como é o propósito da presente revisão.

Sendo assim, uma forma relevante de se categorizar os tipos de riscos pode ser observada na abordagem proposta por Christopher e Peck (2004), a saber: internos à empresa focal (operações, processo e controle); externos para a empresa focal, mas internos para a cadeia (fornecimento e demanda); e externos à cadeia (ataques terroristas, crises mundiais, acidentes naturais, diferenças culturais, por exemplo). Desta forma, tendo em vista que a categorização dos riscos é essencial para se avaliar a abrangência dos modelos de gestão, optou-se por agrupar as publicações selecionadas segundo a abordagem proposta por Christopher e Peck (2004), a qual pode ser observada na Tabela 2.

De maneira geral, as cadeias de suprimentos estão constantemente expostas a um conjunto de riscos que podem gerar distintas formas de perdas, tanto financeiras como de recursos físicos e humanos, ou materiais e de imagem. Entretanto, é possível notar que os riscos que mais impactam as cadeias de suprimentos estão relacionados ao ambiente interno (DANI, 2009).

Corroborando com esta perspectiva, a presente revisão evidencia que a maioria dos trabalhos publicados até o momento foca os riscos que envolvem o ambiente interno das cadeias de suprimentos, 
Tabela 2. Categorização dos tipos de riscos.

\begin{tabular}{clll}
\hline \multicolumn{1}{c}{ Categorias } & \multicolumn{1}{c}{ Artigos* } & \multicolumn{1}{c}{ Subcategorias } & \multicolumn{1}{c}{ Artigos** } \\
\hline Interno à Empresa & $48,52,60,68,73,74,87,90,95$, & Operações e Processo & $27,93$. \\
& $96,99$. & Controle & $46,84,69$. \\
Interno à Cadeia & $1,2,4,5,8,14,15,17,21,23,25,26$, & Demanda & \\
& $28,30,33,34,35,36,39,40,41,42$, & & $12,22,54$. \\
& $43,47,53,55,56,58,62,63,66,70$, & Fornecimento & $3,20,24,31,37,38,50$, \\
& $75,76,78,79,80,81,82,83,86,88$, & & $51,57,59,64,67,71$, \\
& $89,92,94,100$. & Fornecedores e & $77,85,102,103$. \\
& & Clientes*** & $9,10,11,18,19,29$. \\
Externo à Cadeia & $32,72,97$. & Ataques terroristas & 6. \\
& & Crises mundiais & 98. \\
& & Desastres naturais & $16,65$. \\
& & Diferenças culturais & 91. \\
\hline
\end{tabular}

*número de classificação conforme Tabela 1, os autores não mencionam uma única subcategoria. **número de classificação conforme Tabela 1, subcategoria mencionada pelos autores. ***inclusão nossa, essa subcategoria trata dos dois elos em conjunto.

desde o primeiro fornecedor até o consumidor final. Sob esta perspectiva, os riscos referentes ao fornecimento têm sido os mais abordados por tais pesquisas. A busca por estratégias visando melhorias do processo de fornecimento, principalmente no que diz respeito a reduzir interrupções e custos, são as questões mais focadas nesta subcategoria (CAVINATO, 2004; TANG, 2006a; TOMLIN, 2006; ZSIDISIN; PANELLI; UPTON, 2000; ZSIDISIN, 2003; ZSIDISIN; ELLRAM, 2003; ZSIDISIN; SMITH, 2009). Ainda neste contexto, cabe ressaltar os trabalhos de Svensson (2002a, b, c, d, 2003a, b), os quais destacam a importância da colaboração entre empresas como forma de diminuir as vulnerabilidades, tanto no fornecimento de recursos como no nível de serviço aos clientes.

Ademais, outros riscos que envolvem o ambiente interno das cadeias de suprimentos estão relacionados com: tecnologias de informação (LEE, 2002; FAISAL; BANWET; SHANKAR, 2007), falta de flexibilidade/agilidade para atender à demanda (CHRISTOPHER, 2000; RICE; CAINATO, 2003; SHEFFI; RICE, 2005; SKIPPER; HANNA, 2009); falta de confiança/cooperação (LEE; CHRISTOPHER, 2001; HALLIKAS et al., 2004; PECK, 2006; LAEEQUDDIN et al., 2009); falhas operacionais e/ou gerenciais (CHOPRA; SODHI, 2004; BLACKHURST et al., 2005; HALLIKAS; VARIS, 2009; CHRISTOPHER et al., 2011); falta de treinamento (SMITH, 2009); e aspectos financeiros e de mercado (HENDRICKS; SINGHAL, 2003, 2005; BRINDLEY, 2004; FAISAL; BANWET; SHANKAR, 2006).

Com relação aos tipos de riscos internos à empresa, entendem-se, como tais, aqueles ligados diretamente às operações e processos de uma empresa (CHRISTOPHER, 2004), sendo que às suas dimensões podem-se incluir as atividades de $\mathrm{P} \& \mathrm{D}$, de planejamento, compartilhamento de informação ou referentes à estrutura organizacional (LIN; ZHOU, 2011).

Sendo assim, a presente revisão evidenciou que entre os riscos do ambiente interno das organizações, na subcategoria "operações", destacam-se problemas com treinamento de pessoal e falta de melhor controle administrativo (OLSON; DASH WU, 2010). Outrossim, no que diz respeito aos riscos referentes à subcategoria "processos", constatou-se que estes estão relacionados com: falta de flexibilidade no processo produtivo (SODHI, 2005; LIU; LIN; HAYES, 2010); dependência de base única de fornecimento (WAGNER; BODE, 2006; FAISAL; BANWET; SHANKAR, 2008); tecnologia de informação inadequada (JÜTTNER; ZIENGENBEIN, 2009); lead time longo no lançamento de novos produtos (KHAN; CHRISTOPHER; BURNES, 2008; LIN; ZHOU, 2011); perdas de pessoas qualificadas (BLOS et al., 2009).

Por último, nota-se que a categoria de riscos externos à cadeia ainda tem poucas pesquisas realizadas, no entanto, começa a receber maior atenção por parte dos pesquisadores devido às crises financeiras mundiais (JÜTTNER; MAKLAN, 2011), às mudanças no clima e aos desastres naturais (MITROFF; ALPASAN, 2003; PERRY, 2007), uma vez que estes fatores têm impactado fortemente as cadeias de suprimentos nos últimos anos (JÜTTNER; MAKLAN, 2011). 


\subsection{Etapas elementares de um modelo de gestão de riscos}

Como exposto antes, a gestão de riscos tem sido um instrumento importante no que diz respeito à busca por uma efetiva redução de vulnerabilidades em cadeias de suprimentos (LIN; ZHOU, 2011). Nesse contexto, alguns pesquisadores têm sugerido modelos que possam servir como diretrizes para a prática dessa gestão.

Entretanto, Zsidisin e Ritchie (2009) alertam que os modelos podem diferenciar-se uns dos outros, em termos de etapas que os compõem. De acordo com Mullai (2009), a variação em termos de etapas que compõem os modelos de gestão de riscos existentes na literatura pode ser atribuída a fatores como: a) influência de diferentes contextos (políticos, econômicos e sociais, por exemplo) nas percepções dos autores acerca dos riscos; ou b) diferentes necessidades (acerca dos riscos) por segmentos distintos, ou seja, cada país ou região tem suas prioridades, assim como cada empresa tem interesses diferenciados, o que acaba, segundo o autor, influenciando a proposição de tais modelos.

Não é o objetivo do presente artigo esgotar o assunto, principalmente no que diz respeito à complexidade de critérios estabelecidos pelos autores na elaboração de tais etapas, e sim elaborar um conjunto de etapas que podem ser consideradas elementares, para se poder, posteriormente, analisar e discutir alguns dos modelos existentes na literatura.

Neste contexto, entende-se que a primeira etapa a compor um modelo de SCRM deva ser a identificação dos riscos. Segundo Zsidisin e Ritchie (2009), nesta etapa, realizam-se as atividades de levantamento das fontes de riscos com caracterização. A identificação dos riscos inicia o processo de gestão de riscos e tem como objetivo descobrir todos os riscos relevantes na cadeia em estudo. Isto implica que um julgamento precoce é necessário para decidir se um risco pode ser considerado relevante - e assim continuar a ser avaliado - ou não. Cabe lembrar que a identificação de riscos deve seguir uma abordagem holística (BUHMAN; KEKRE.; SINGHAL, 2005), com observação ampla da cadeia de abastecimento para identificar pontos fracos, ameaças potenciais e todas as vulnerabilidades relevantes.

Craighead et al. (2007) afirmam que as empresas ou cadeias precisam desenvolver a capacidade de efetuar análises precoces, de modo que os riscos possam ser devidamente avaliados, a fim de que os esforços de mitigação possam ter efeito. Assim, os riscos relevantes podem ser reconhecidos entecipadamente e as ações de mitigação podem ter melhor efeito (CRAIGHEAD et al., 2007; HENDRICKS; SINGHAL, 2003; TOMLIN, 2006; ZSIDISIN et al., 2004).
No entanto, devido à complexidade das operações de uma cadeia de abastecimento, a capacidade de identificação dos riscos requer o conhecimento da empresa sobre os componentes mais críticos no que diz respeito ao seu processo produtivo e fornecedores, a fim de concentrar esforços nas áreas mais vulneráveis de cada operação (HALLIKAS; VIROLAINEN; TUOMINEN, 2002; KLEINDORFER; SAAD, 2005).

A segunda etapa, denominada avaliação dos riscos, consiste em: i) selecionar os riscos mais importantes para a cadeia; e ii) avaliar o impacto destes riscos em termos de ocorrência e potenciais consequências (ZSIDISIN; RITCHIE, 2009). A estas atividades, Rice e Cainato (2003) acrescentam que é necessário estimar a probabilidade de ocorrência de cada risco, bem como avaliar as perdas potenciais.

O principal objetivo da avaliação dos riscos é auxiliar a compreensão dos fatores que levam à ocorrência de um risco específico, ao mesmo tempo que fornece informações sobre o impacto destes, a fim de que se possa evitá-los ou reduzir o efeito de suas consequências por meio de estratégias de contingência (ZSIDISIN et al., 2004).

Além disso, nota-se que a etapa de avaliação dos riscos possibilita uma clara compreensão dos riscos mais relevantes em uma cadeia, oferencendo oportunidade para que sejam colocados em uma ordem de prioridade. Para o processo de avaliação ser eficaz, esta etapa necessita ser simples e pode ser auxiliada com ilustrações gráficas para mapear os riscos e, de maneira adequada, mostrar onde, quando e com que probabilidade e impacto eles podem ocorrer (HALLIKAS; VIROLAINEN; TUOMINEN, 2002; HARLAND, BRENCHLEY; WALKER, 2003; MANUJ; MENTZER, 2008b; MATOOK; LASCH; TAMASCHKE, 2009; RITCHIE; BRINDLEY, 2007).

A terceira etapa do processo de gestão é denominada controle dos riscos e consiste em estabelecer propostas (ZSIDISIN; RITCHIE, 2009) ou estratégias (RICE; CAINATO, 2003) para mitigação dos riscos. As atividades de mitigação fazem uso dos dados coletados na etapa anterior para enfrentar os riscos potenciais com ações corretas. Isto inclui estratégias de mitigação (antes do evento de risco acontecer), bem como planos de contingência (após o evento de risco ter ocorrido). Para cada risco relevante, uma estratégia apropriada de mitigação precisa ser desenvolvida e executada (CHOPRA; SODHI, 2004; KLEINDORFER; SAAD, 2005; MANUJ; MENTZER, 2008b; WAGNER; BODE, 2006).

Kleindorfer e Saad (2005, p. 15) argumentam que, em se tratando de gestão de riscos, é melhor a prevenção do que a cura ("prevention is better than cure"), ou seja, é melhor planejar e realizar a gestão de riscos, do que sofrer os efeitos negativos de sua materialização. Sob esta perspectiva, a literatura sugere que uma mitigação eficaz dos riscos só pode ser alcançada por 
meio de estreita colaboração entre os parceiros da cadeia de abastecimento (KLEINDORFER; SAAD, 2005; ZSIDISIN et al., 2004).

Com relação à última etapa, é composta pelo monitoramento dos riscos e avaliação do impacto da mitigação dos riscos sobre os resultados de desempenho (ZSIDISIN; RITCHIE, 2009). Com relação ao monitoramento, Mullai (2009) destaca duas possibilidades, de medidas: i) eficácia, que é o resultado da eliminação ou mitigação dos riscos em relação às ações propostas na terceira etapa; e ii) eficiência das ações propostas, que pode ser medida pela perda financeira (caso nenhuma ação fosse tomada) em relação ao gasto financeiro evitado pela contenção do risco. A respeito do impacto da mitigação dos riscos sobre os resultados de desempenho, Ritchie e Brindley (2007) lembram que podem ser usadas em sua avaliação, tanto medidas financeiras como não financeiras. A Figura 3 mostra as etapas discutidas até aqui.

\section{Discussão dos resultados}

\subsection{Análise dos modelos pesquisados}

Inicialmente, cabe destacar que a gestão de riscos em cadeias de suprimentos é um processo complexo e permeado por variáveis de difícil quantificação (SVENSSON, 2000). Neste contexto, considera-se que um modelo universalmente aplicável, completo e confiável para a análise e gestão dos riscos em cadeias de suprimentos, ainda não foi desenvolvido
(AGUIAR, 2010). Mesmo assim, a presente pesquisa bibliográfica possibilitou encontrar alguns modelos interessantes, os quais serão analisados segundo a abordagem proposta para o presente trabalho, tal como demonstra a Tabela 3.

A respeito dos modelos pesquisados, nota-se que, de um total de seis modelos selecionados, quatro podem ser considerados os mais completos, em termos de alcance aos tipos de riscos e etapas que os compõem. Além disso, optou-se por excluir da análise o modelo Hallikas, Virolainen e Tuominen, (2002), devido ao fato de que este modelo, segundo seus autores, foi elaborado com foco em pequenas e médias empresas; e optou-se por excluir também o modelo Wu, Blackhurst e Chidambaram (2006), porque, segundo seus autores, este faz parte de um aplicativo computadorizado. A justificativa para tal exclusão refere-se ao fato de que estes dois modelos (HALLIKAS; VIROLAINEN; TUOMINEN, 2002; WU et al., 2006) distoam do propósito da presente revisão, qual seja, um modelo de aplicação mais abrangente, sem ser específico para um determinado segmento ou setor. Sendo assim, inicia-se uma análise pormenorizada dos quatro modelos restantes a fim de possibilitar um melhor entendimento dos elementos que os compõem, bem como de suas etapas de aplicação.

\subsubsection{O modelo de Harland, Brenchley e Walker (2003)}

Este modelo foi desenvolvido a partir de observações realizadas pelos autores acerca das

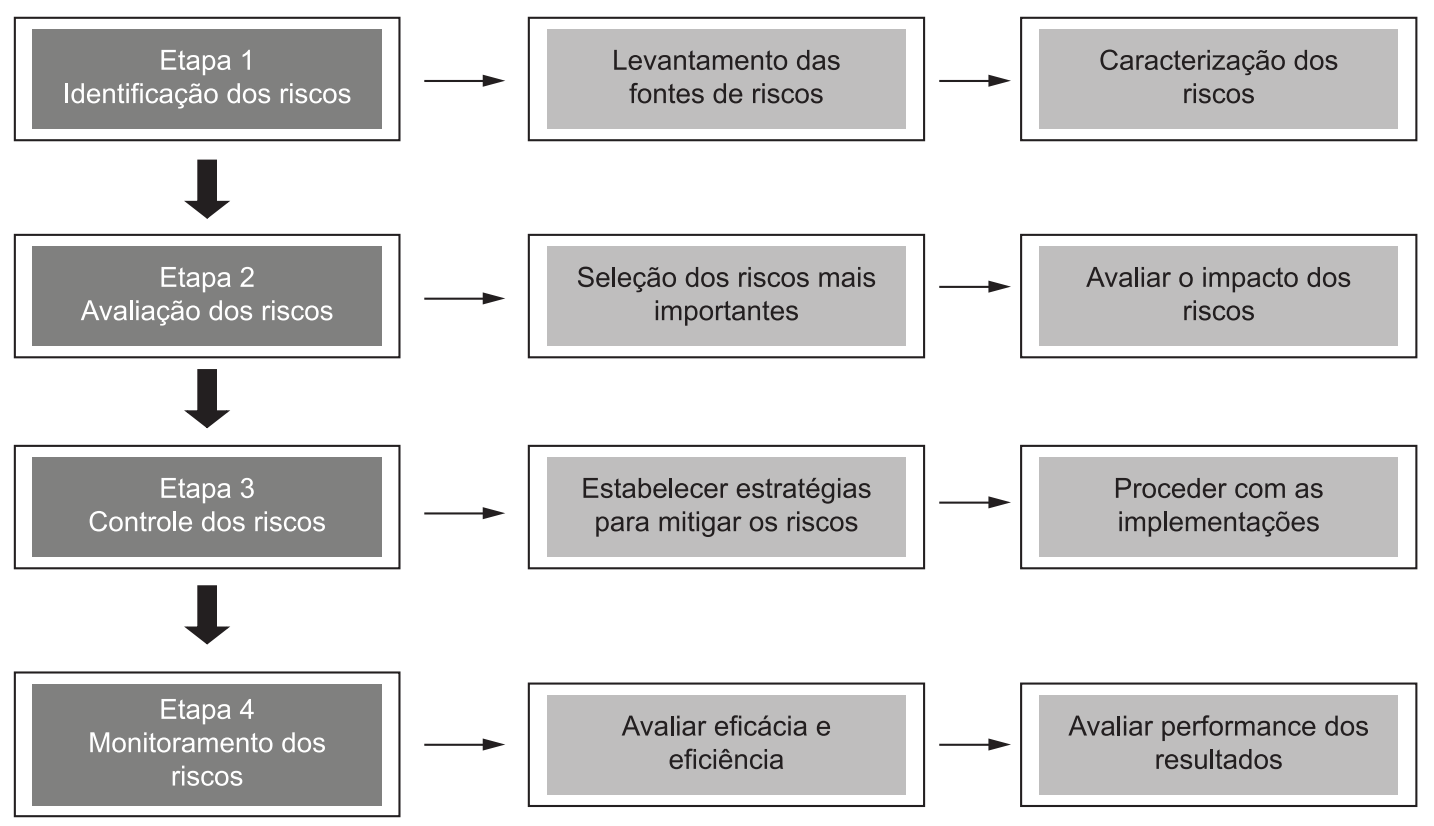

Figura 3. Etapas elementares para a gestão de riscos. Fonte: Elaborado com base em Hallikas, Virolainen e Tuominen, (2002), Harland, Brenchley e Walker (2003), Kleindorfer e Saad (2005), Wu, Blackhurst e Chidambaram (2006) e Tummala e Schoenherr (2011). 
Tabela 3. Análise dos modelos.

\begin{tabular}{|c|c|c|c|c|c|c|c|c|c|}
\hline \multirow{3}{*}{ Autores } & \multirow{3}{*}{ Ano } & \multicolumn{8}{|c|}{ Critérios para análise dos Modelos } \\
\hline & & \multicolumn{5}{|c|}{ Etapas que os compõem } & \multicolumn{3}{|c|}{$\begin{array}{l}\text { Capacidade de } \\
\text { abrangência }\end{array}$} \\
\hline & & I & II & III & IV & MFA* & IE & IC & EC \\
\hline Hallikas, Virolainen e Tuominen, & 2002 & $\mathbf{X}$ & $\mathbf{X}$ & - & - & Não & - & $\mathbf{X}$ & - \\
\hline Harland, Brenchley e Walker & 2003 & $\mathbf{X}$ & $\mathbf{X}$ & $\mathbf{X}$ & - & Sim & - & $\mathbf{X}$ & - \\
\hline Kleindorfer e Saad & 2005 & $\mathbf{X}$ & $\mathbf{X}$ & $\mathbf{X}$ & - & Não & - & $\mathbf{X}$ & $\mathbf{X}$ \\
\hline Peck & 2005 & $\mathbf{X}$ & $\mathbf{X}$ & - & - & Não & $\mathbf{X}$ & $\mathbf{X}$ & $\mathbf{X}$ \\
\hline Tummala e Schoenherr & 2011 & $\mathbf{X}$ & $\mathbf{X}$ & $\mathbf{X}$ & $\mathbf{X}$ & Não & $\mathbf{X}$ & $\mathbf{X}$ & - \\
\hline Wu, Blackhurst e Chidambaram & 2006 & $\mathbf{X}$ & $\mathbf{X}$ & - & - & Sim & - & $\mathbf{X}$ & $\mathbf{X}$ \\
\hline
\end{tabular}

*MFA = modelo foi aplicado em estudo de caso? I "Identificação dos Riscos", II "Avaliação dos Riscos", III

"Controle dos Riscos", IV “Monitoramento". IE "Interna à Empresa”, IC "Interna à Cadeia”, EC "Externa à Cadeia”.

crescentes complexidades que afetam as cadeias de suprimentos. Depois de uma bem elaborada revisão da literatura, os autores identificaram onze tipos de riscos e seis tipos de perdas comuns que afetam as cadeias de suprimentos. A partir desta revisão, os autores propuseram um modelo para gestão de riscos composto de seis etapas, tal como mostra a Figura 4. Posteriormente, os autores aplicaram o referido modelo em quatro estudos de caso envolvendo indústrias de componentes eletrônicos, nos Estados Unidos e no Reino Unido. Cabe ressaltar que este é um dos poucos casos, na literatura sobre gestão de riscos em cadeias de suprimentos, em que os autores submetem um modelo teórico à aplicação prática, a fim de testá-lo, para, em seguida, discutir sua aplicabilidade.

De modo geral as seis etapas apresentadas no referido modelo são equivalentes às três primeiras etapas elementares do modelo apresentado por esta revisão (Figura 3), sendo que, neste caso, os autores não avançam na questão de monitoramento dos riscos, ou seja, o modelo apresentado por Harland, Brenchley e Walker (2003) encerra-se com a fase de implementação das estratégias para mitigar os riscos.

Como pontos de incremento deste modelo, destacam-se o "mapeamento de riscos na cadeia" como início do processo de gestão, e a inclusão da colaboração na etapa 5 , como forma de destacar a necessidade de elaboração conjunta na estratégia de mitigação dos riscos da rede. Além disso, a aplicação do modelo em casos reais possibilitou, segundo os autores, a compreensão de que, na prática, a disponibilidade de dados que permite avaliar a probabilidade de ocorrência dos riscos nem sempre pode ser satisfatória e de fácil acesso.

Outro ponto importante na aplicação do modelo refere-se ao fato de que os pesquisadores consideraram difícil a elaboração de métricas capazes de quantificar os riscos.

\subsubsection{O modelo de Kleindorfer e Saad (2005)}

Diferentemente do modelo apresentado na seção anterior - o qual tinha como foco de análise,

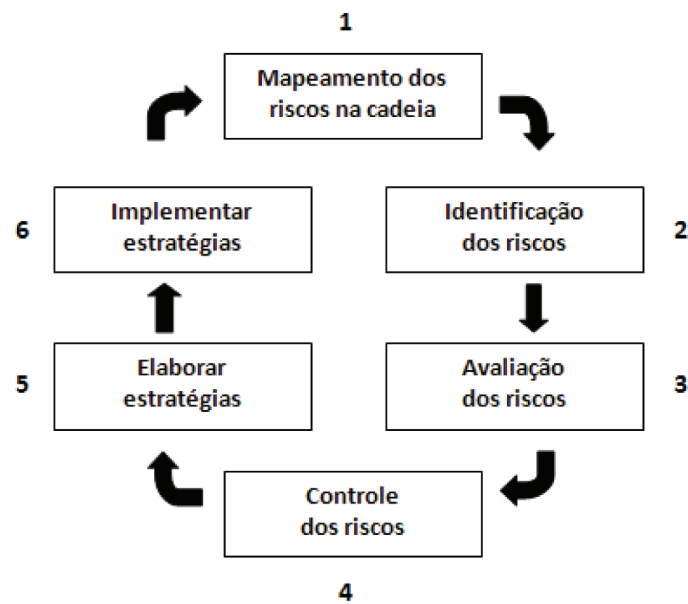

Figura 4. O modelo de Harland, Brenchley e Walker (2003). Fonte: Adaptado dos mesmos autores.

exclusivamente, o ambiente interno de uma cadeia de suprimentos -, no modelo apresentado por Kleindorfer e Saad (2005), amplia-se o foco de análise para além do ambiente interno das cadeias, contextualizando, também, fatores gerados no ambiente externo, os quais, segundo os autores, são passíveis de provocar perdas para a cadeia como um todo.

Este modelo foi elaborado a partir de dados empíricos coletados em indústrias químicas norteamericanas. Apoiados nos dados empíricos e em uma revisão de literatura, os autores propuseram um modelo de análise baseado em três fases, denominadas de SAM (Specifying sources of risk and vulnerabilities, Assestement, and Mitigation), que são equivalentes às três fases iniciais do modelo apresentado na Figura 3 , ou seja, identificação, avaliação e controle dos riscos. A Figura 5 demonstra o modelo discutido nesta seção.

Ao incorporar os fatores de riscos oriundos do ambiente externo, os autores fazem deste modelo uma referência para a prática mais abrangente da gestão de riscos em cadeias de suprimentos, uma vez que, até então, pouco se discutia sobre a presença deste tipo de riscos nas cadeias de suprimentos. Dessa 
forma, os autores demonstram empiricamente que os riscos advindos de fatores externos são tão danosos à eficiência das cadeias de suprimentos quanto os riscos relacionados ao ambiente interno. Entretanto, este modelo, também não avança nas discussões acerca do monitoramento dos riscos, sendo esta, uma limitação ressaltada pelos autores.

\subsubsection{O modelo de Peck (2005)}

Este modelo pode ser considerado o mais amplo em termos de capacidade de abrangência dos tipos de riscos, uma vez que aborda as fontes e os direcionadores de vulnerabilidades, tendo como foco o alcance horinzontal e vertical dos riscos sob a perspectiva de quatro níveis de análise. O modelo de Peck (2005) foi elaborado a partir de um estudo de caso exploratório envolvendo cadeias de suprimento da indústria aeroespacial britânica, e validado por meio de entrevistas em profundidade com gestores representando outros setores da economia do Reino Unido. A Figura 6 demonstra o modelo em questão.

No nível 1, os riscos da cadeia de suprimentos podem ser analisados a partir dos processos de negócios. Tal abordagem aspira a um fluxo perfeito

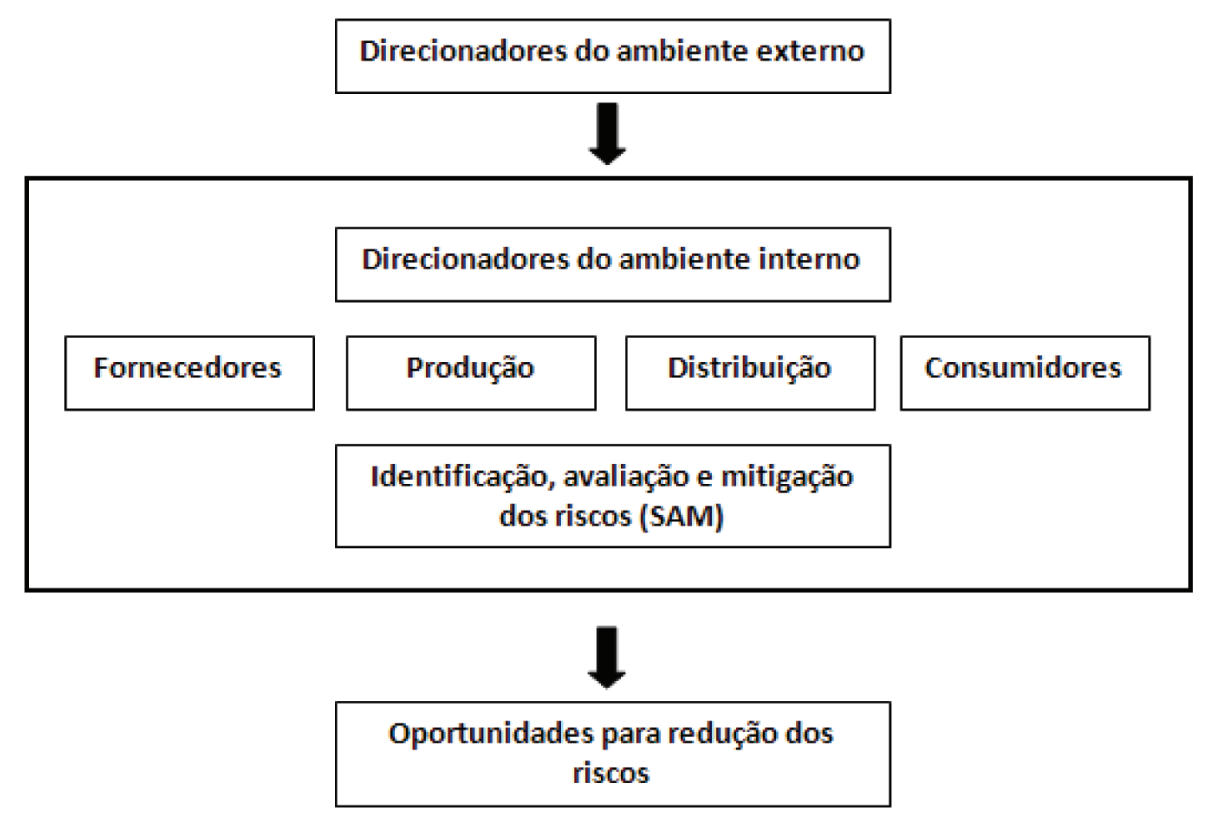

Figura 5. O modelo de Kleindorfer e Saad (2005). Fonte: Adaptado dos mesmos autores.

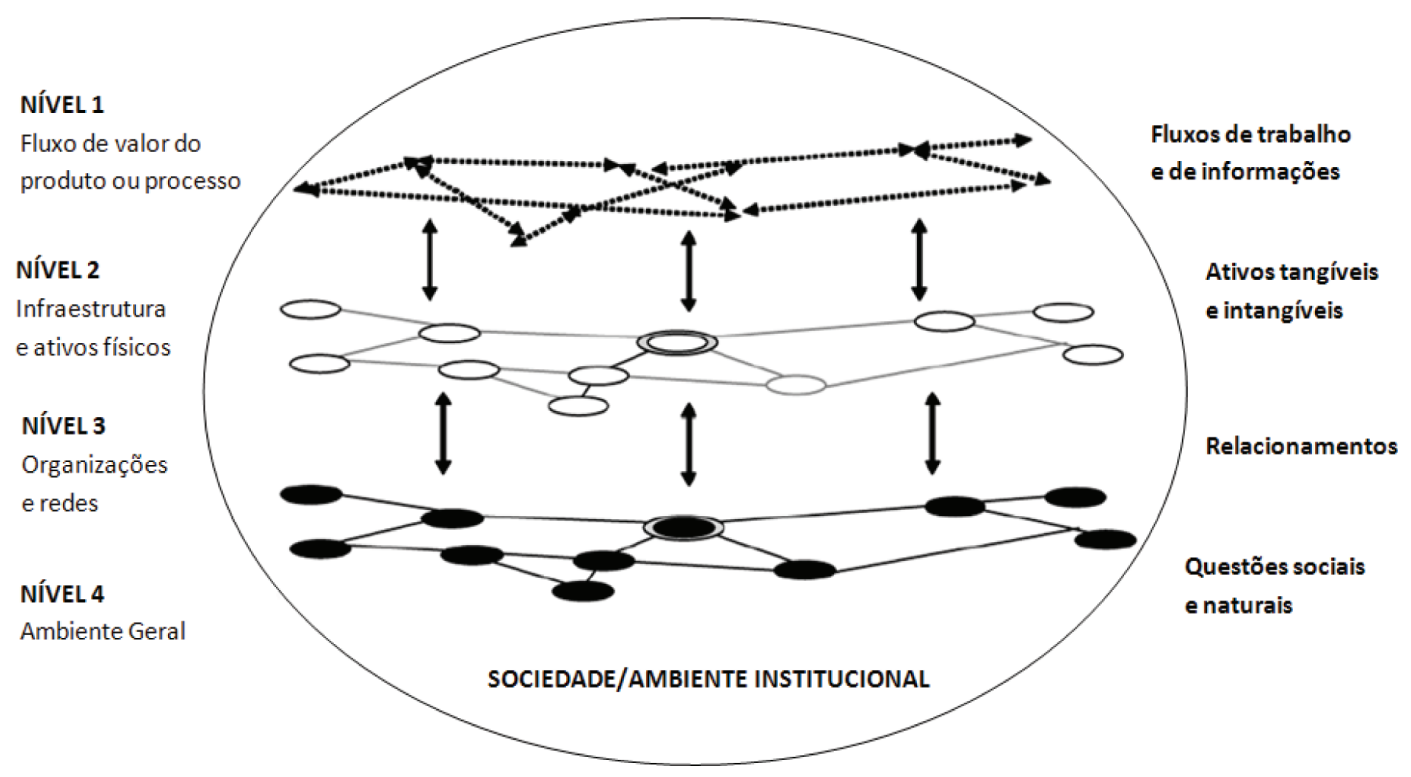

Figura 6. O modelo de Peck (2005). Fonte: Adaptado da mesma autora. 
de informações e de materiais por toda a cadeia de abastecimento de forma a levar todos os parceiros a agirem de forma coordenada. Neste nível, as cadeias de abastecimento são vistas em termos do conteúdo como um "alinhamento logístico" fluindo entre os elos da cadeia.

Sob esta perspectiva, a ênfase do modelo está baseada na eficiência, no valor, desenvolvimento e gestão dos processos relativos aos fluxos de trabalho $\mathrm{e}$ as informações que os acompanham - geralmente por produto ou classe de produto. Dessa forma, a autora ressalta que, neste nível, os riscos são principalmente as consequências financeiras ou comerciais de ineficiências ou subótimo referente ao desempenho da cadeia de suprimentos, incluindo a incapacidade de reagir rapidamente à volatilidade da demanda e às mudanças de mercado.

O nível 2 de análise aborda as cadeias de fornecimento em termos de bens e infraestrutura necessários para produzir e transportar as mercadorias, bem como para suportar os fluxos de informação elencados no nível 1 . Sob esta perspectiva, a autora afirma que os pontos de contato entre os elos são ativos comerciais, locacionais ou instalações (por exemplo, campos, fábricas, centros de distribuição, varejo). A este nível acrescenta-se a resiliência da rede que deve ser avaliada em termos de perdas de ligações, nós e outros ativos operacionais essenciais, por exemplo, a mão de obra qualificada.

Este nível também evidencia que a distribuição física entre os elos e para o consumidor final continua a ser um elemento essencial da cadeia de suprimentos integrada. Assim, interrupções de transporte são vistas como uma fonte potencial de vulnerabilidade para todos os membros da cadeia (McKINNON, 2004).

Em termos de impacto sobre o desempenho do nível 1, tais vulnerabilidades podem ser classificadas em três grandes grupos: perdas, danos e atrasos (CHRISTOPHER; LEE, 2001). Além disso, perdas, danos e atrasos podem impactar os níveis de serviço, com os dois primeiros também causando discrepâncias em demanda e disponibilidade de dados para ações (PECK, 2006).

No nível 3, as cadeias de suprimentos são vistas sob a perspectiva de redes interorganizacionais. Assim, nesse nível os riscos movem-se desde o fornecimento até o patamar da gestão corporativa, estratégia de negócios e microeconomia. Aqui os pontos de contato entre os elos são as organizações - setor comercial e público - às quais cabe possuir ou gerir o patrimônio e infraestrutura, por meio das quais fluem os bens físicos e fluxos de informações. Os ligamentos entre os elos se dão pelas relações comerciais, especialmente as dependências de poder entre organizações. Cabe mencionar, ainda, que no nível 3 surge a premissa de que organizações fortes irão abusar da sua posição de poder diante dos membros mais fracos.
Por fim, o quarto e último nível é o ambiente mais amplo ligado aos determinantes macroeconômicos e naturais, dentro dos quais as organizações fazem negócios, posicionam bens e infraestrutura. Fatores para análise são os políticos, econômicos, sociais e tecnológicos, elementos do ambiente operacional (incluindo questões jurídicas e regulamentares) bem como fenômenos naturais, geológicos, meteorológicos ou patológicos.

É importante ressaltar que todos estes fatores podem afetar a cadeia de suprimentos em cada um dos três primeiros níveis da estrutura. Não obstante, os riscos que emanam deste nível são susceptíveis de estar fora do controle direto dos gestores.

\subsubsection{O modelo de Tummala e Schoenherr (2011)}

Tummala e Schoenherr (2011) apresentam, de maneira detalhada, aquele que pode ser considerado, segundo o propósito estabelecido por esta revisão, como o modelo mais completo e mais bem estruturado, em termos de etapas para a gestão de risco em cadeias de suprimentos. Este modelo está divido em três fases e sete etapas, conforme demonstra a Figura 7.

A fase 1 deste modelo é composta pela identificação dos riscos potenciais e pela determinação das consequências de todos os riscos potenciais. Os autores sugerem que, nesta fase, os riscos potenciais sejam classificados segundo seu "grau de severidade", que, neste caso, podem ser: catastrófico, crítico, marginal ou desprezível. Ainda nesta fase, ocorre a avaliação dos riscos, sendo que cabe aos gestores, por meio desta avaliação, determinar a frequência com que cada risco poderá ocorrer. Os autores sugerem que, para cada risco (após ser estabelecido o grau de severidade), seja estimada a probabilidade de ocorrência, que, neste caso, poderá ser: muito frequente (uma vez por semana); frequente (uma vez por mês); rara (uma vez por ano); e extremamente rara (uma vez a cada dez anos).

$\mathrm{Na}$ fase 2, deverão ocorrer o controle e a mitigação dos riscos. Para iniciar a etapa de controle, os autores sugerem que seja elaborado um ranqueamento dos riscos mais importantes para a cadeia, segundo o grau de severidade e a probabilidade de ocorrência. Depois do ranqueamento, os riscos deverão ser separados em três categorias, a saber: inaceitáveis, toleráveis e aceitáveis. Em seguida, um plano de ação deverá ser elaborado visando à mitigação de cada risco considerado inaceitável ou tolerável. Ainda nesta fase, os autores consideram que os gestores devem realizar uma avaliação financeira sobre o custo de cada ação de mitigação. Sendo assim, recomendam uma classificação para os riscos segundo o valor de sua possível mitigação, por exemplo: substancial (mais de $\$ 100.000)$; média (entre $\$ 10.000$ e \$100.000); 
baixa (entre $\$ 1.000$ e $\$ 10.000$ ); e trivial (menos do que \$1.000).

Na fase 3, para o monitoramento dos riscos, os autores afirmam que os gestores poderão recorrer a ações corretivas e avaliação do desempenho. Caso os resultados esperados não sejam alcançados, uma tomada de decisão poderá lançar novas diretivas, fazendo com que o processo de gestão de riscos retorne à Fase 1. Ademais, para auxiliar o monitoramento dos riscos, os autores destacam como possibilidade a utilização de programas de computadores, por exemplo, o Software as a Service (SaaS).

\subsubsection{A proposta do modelo final}

Como destacado anteriormente, a proposição de um modelo para gestão de riscos em cadeias de suprimentos sofre influência de diversos fatores. Além disso, entende-se que sua elaboração é apenas uma das partes que deverão compor a estratégia de gestão

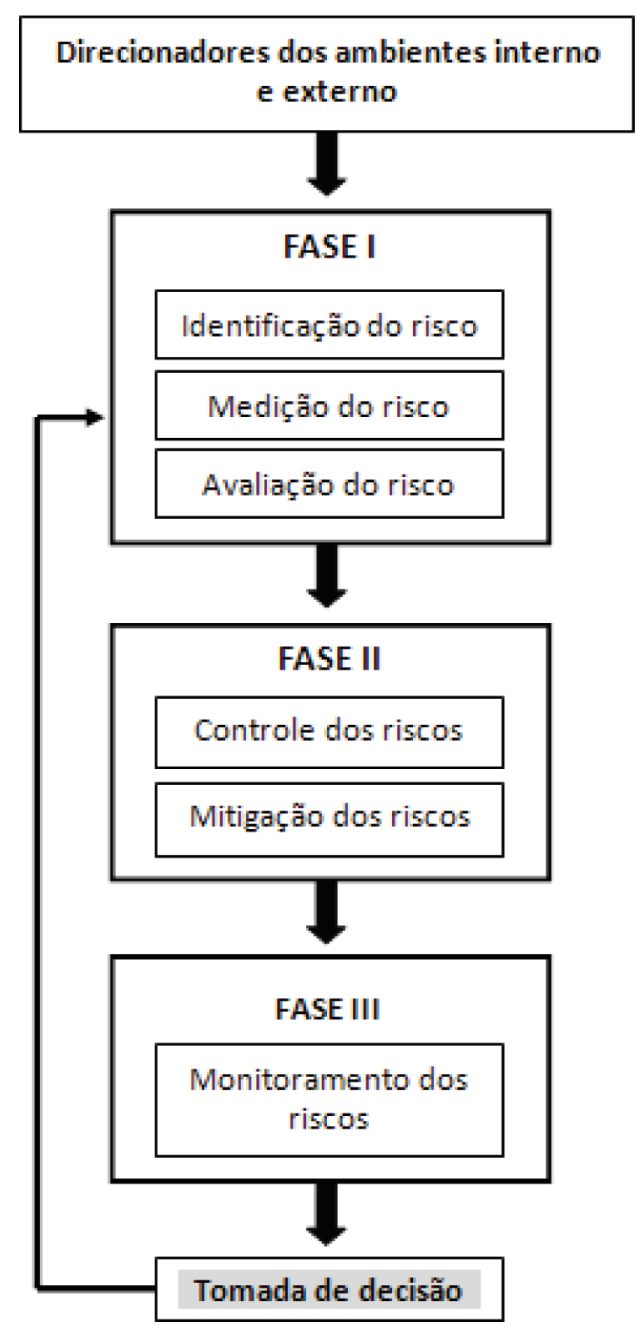

Figura 7. O modelo de Tummala e Schoenherr (2011). Fonte: Adaptado dos mesmos autores. de riscos em cadeias de suprimentos (DANI, 2009). Neste caso, tal estratégia poderá assumir uma posição proativa ou reativa aos eventos de riscos (NORRMAN; JANSSON, 2004).

A este respeito, Dani (2009) assevera que uma estratégia visando à gestão de riscos é reativa quando uma cadeia de suprimentos opera sem se preocupar com os riscos, reagindo para mitigar os efeitos somente no momento de sua materialização. Em sentido oposto, o mesmo autor ressalta que a estratégia proativa é adotada quando os riscos potenciais de uma cadeia de suprimentos são identificados, e ranqueados em termos de importância - resultado da probabilidade de ocorrência e possíveis consequências - e, posteriormente, mitigados. $\mathrm{O}$ autor lembra ainda que, em determinados casos, é difícil propor a mitigação dos riscos, sendo que para tais casos, existe a necessidade de se desenvolver e implementar planos de contingências visando diminuir o impacto dos riscos, quando estes ocorrerem.

É importante destacar que um modelo proativo para gestão de riscos é o que se busca na presente revisão, especificamente, um modelo que seja abrangente o suficiente para identificar e controlar o maior número possível de riscos e vulnerabilidades em cadeias de suprimentos. Entretanto, como visto anteriormente, parte significativa dos modelos existentes na literatura possui limitações com relação à sua capacidade de abrangência.

Desse modo, uma alternativa coerente com o propósito do presente estudo seria a junção dos dois modelos considerados mais completos (segundo os resultados apresentados na Tabela 3) em apenas um, uma vez que os riscos existem tanto dentro das empresas como interna e externamente às suas respectivas cadeias de suprimentos, com impactos distintos (GAUDENZI, 2009). A Figura 8 ilustra a junção entre as etapas de gestão propostas por Tummala e Schoenherr (2011) e os níveis de análise apresentados no modelo de Peck (2005), formando, no entendimento dos autores do presente estudo, o modelo ideal para análise e gestão de riscos em cadeias de suprimentos.

\subsection{Elementos que podem ser acrescentados ao modelo final}

Como destacado anteriormente, a aplicação prática de um modelo é um importante componente no seu processo de aprimoramento (HARLAND; BRENCHLEY; WALKER, 2003). Entretanto, no que diz respeito ao modelo apresentado por esta revisão (Figura 8), nota-se que, mesmo antes de sua aplicação prática, algumas melhorias podem ser realizadas.

Assim, o primeiro elemento a ser acrescentado ao modelo final refere-se ao que pode ser denominado direcionadores dos ambientes interno e externo, 


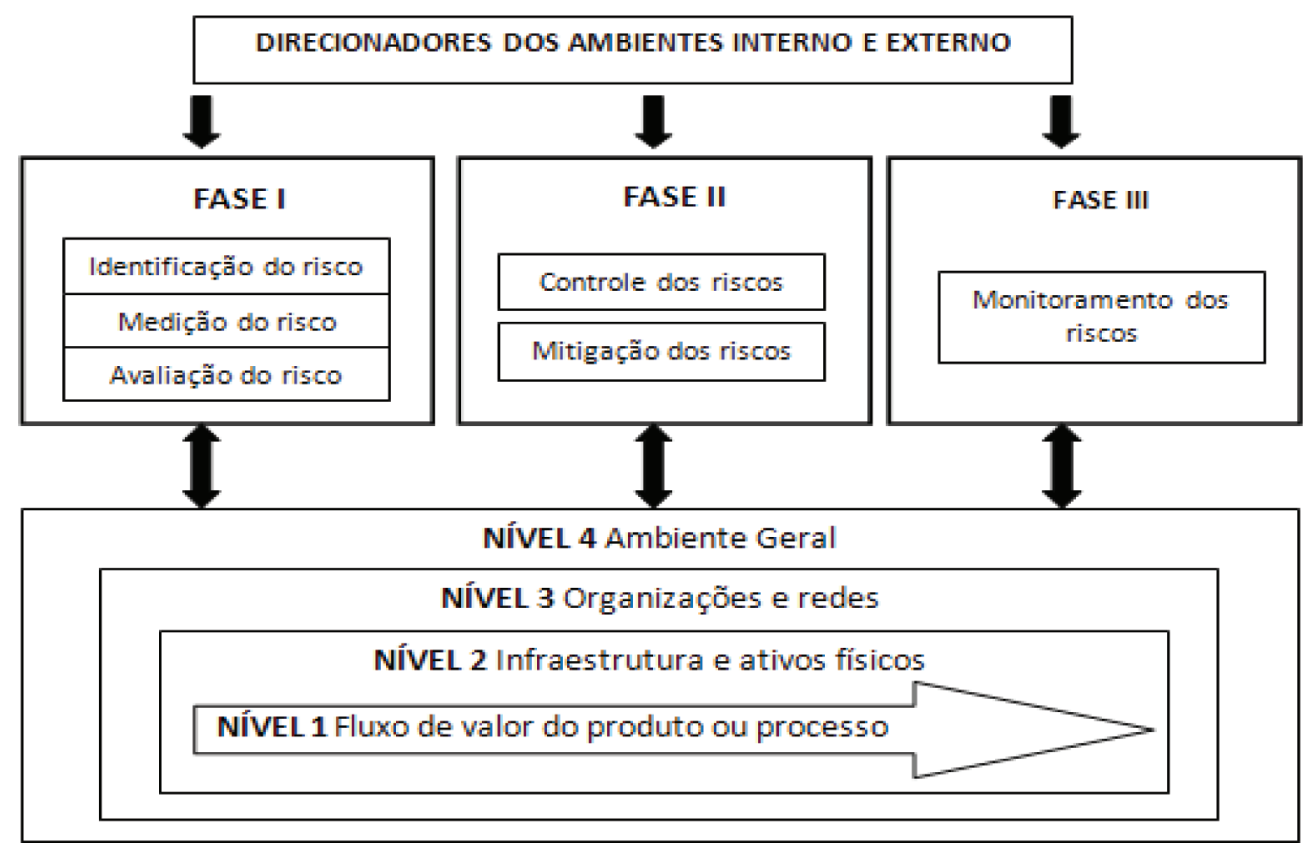

Figura 8. O modelo final. Fonte: Elaborado pelos autores a partir de Tummala e Schoenherr (2011) e Peck (2005).

uma vez que, no modelo proposto por Tummala e Schoenherr (2011), não é apresentada uma forma estruturada para selecionar e organizar estes direcionadores. Isto representa um risco grave à eficiência do modelo, pois se entende que os direcionadores são essenciais para a identificação de vulnerabilidades (ASBJORNSLEET, 2009), sendo que sua escolha, caso não seja feita de maneira adequada, poderá afetar significativamente os resultados do processo de gestão (GAUDENZI, 2009).

Asbjornsleet (2009) sugere, sob a perspectiva de um diagrama, uma forma para se estruturar um amplo conjunto de direcionadores internos e externos de riscos em cadeias de suprimentos, evitando-se perda de informação relenvante na etapa de identificação. Assim, entende-se que esta forma de selecionar e organizar os direcionadores poderá ser incorporada ao modelo final. A Figura 9 apresenta a estrutura em questão.

Outro elemento que pode ser acrescentado ao modelo final refere-se a uma atividade que deverá ser realizada na fase de monitoramento dos riscos, aqui denominada como comunicação dos riscos. Segundo Mullai (2009), após a finalização de um processo de gestão de riscos em cadeias de suprimentos, não raramente, ocorrem efeitos secundários oriundos das decisões tomadas para mitigação dos riscos, sendo que estes efeitos secundários (que antes do processo de gestão dos riscos não eram conhecidos ou nem existiam) podem trazer consequências para outras cadeias e para a sociedade como um todo. Por isso, se faz importante sua comunicação a todas as partes interessadas (MULLAI, 2009).

\subsection{Perspectivas para futuras pesquisas}

No cenário internacional, existem indícios de que as pesquisas envolvendo modelos para gestão de riscos em cadeias de suprimentos devem seguir três perspectivas diferentes, a saber: i) modelos que contemplem a gestão de riscos em cadeias globais (KOUVELIS et al., 2011); ii) modelos com inclusão de métodos quantitativos para análise de riscos (TANG, 2006a; MELNYK; RODRIGUES; RAGATZ, 2009); e iii) modelos capazes de analisar com maior precisão o impacto dos riscos no desempenho das cadeias (AGUIAR, 2010). Ainda com relação ao cenário internacional, cabe destacar um fluxo crescente de pesquisas sobre gestão de riscos em cadeias de suprimentos realizadas por países como China, Índia e Malásia.

No que diz respeito ao contexto brasileiro de pesquisa, como mencionado antes, a temática envolvendo a gestão de riscos em cadeias de suprimentos ainda é um campo quase totalmente inexplorado. Entende-se que existe um amplo conjunto de possibilidades de análises, desde pesquisas que tragam conhecimentos sobre os tipos de riscos que afetam as cadeias produtivas brasileiras até pesquisas mais complexas que busquem alternativas de mitigação ou eliminação.

Ainda com relação ao contexto brasileiro, nota-se, atualmente, uma preocupação de diversos atores (tanto públicos como da iniciativa privada) no que 


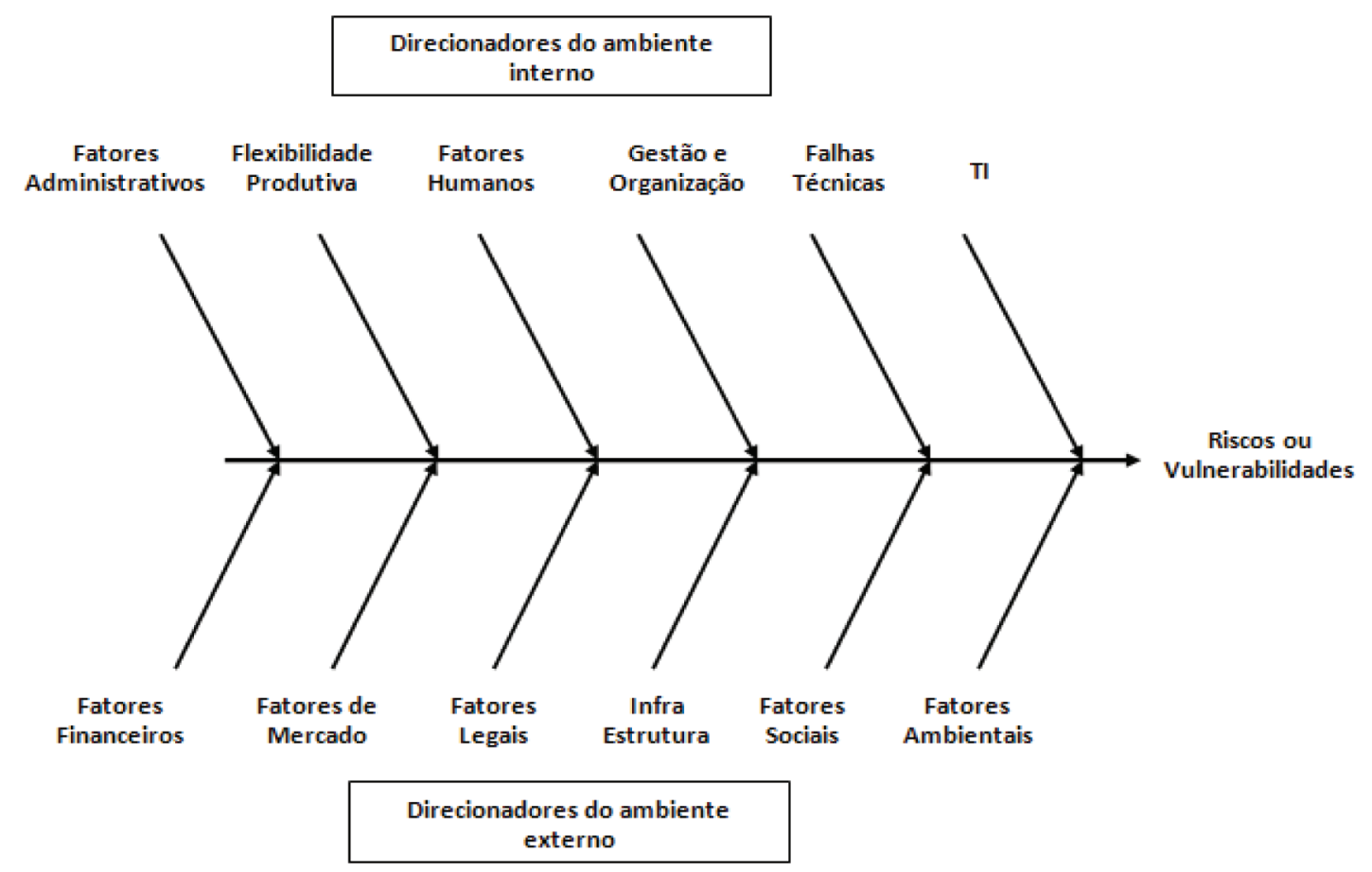

Figura 9. Estrutura para selecionar e organizar os direcionadores. Fonte: Adaptado de Asbjornsleet (2009).

tange a melhorar a eficiência de alguns setores produtivos do País, seja por meio de investimentos em tecnologia e inovação ou por meio de ações gerenciais visando ao aumento de desempenho das empresas. Neste contexto, pesquisas envolvendo a gestão de riscos em cadeias brasileiras podem oferecer uma contribuição significativa, tanto para as cadeias produtivas que atendem ao mercado interno como para aquelas que competem em mercados internacionais, tais como, cadeias de produtos do agronegócio, de petróleo, de aviões civis e militares (caso da Embraer), de bio combustíveis, da indústria automotiva, entre outras.

\section{Considerações finais}

Os atuais padrões mundiais de concorrência têm pressionado as empresas a se tornarem cada vez mais competitivas, obrigando-as a reverem suas formas de operação a fim de reduzir custos e gerar mais receitas. Dentro deste contexto, surge o conhecimento de que a eficiência de uma empresa não depende somente de suas operações internas, mas também da capacidade de uma coordenação mais próxima com seus fornecedores e distribuidores, capturando as sinergias das interações entre as firmas que compõem uma cadeia de suprimentos (LAMBERT; POHLEN, 2001; MENTZER et al., 2001).

A abordagem envolvendo riscos na gestão da cadeia de suprimentos tem sido objeto de estudo de várias pesquisas ao redor do mundo, contudo, no Brasil, a temática envolvendo a gestão de riscos em cadeias de suprimentos ainda é um campo quase totalmente inexplorado. Dessa forma, a presente revisão objetivou auxiliar um melhor conhecimento no que se refere aos riscos que podem incidir sobre uma cadeia de suprimentos e os modelos mais adequados para geri-los. Para tanto, foram revisados 103 estudos científicos publicados entre 2000 e 2012, com o propósito de categorizar os tipos de riscos abordados nos artigos científicos segundo a descrição proposta por Christopher e Peck (2004), e analisar os modelos disponíveis na literatura para a prática dessa gestão.

Como parte dos resultados alcançados, constatou-se que parte significativa dos modelos existentes na literatura possui limitações com relação à sua capacidade de abrangência. Assim, uma alternativa coerente com o propósito do presente estudo foi a junção dos dois modelos considerados mais completos, a saber, Tummala e Schoenherr (2011) e Peck (2005), formando um modelo proativo para gestão de riscos, abrangente o suficiente para identificar e controlar o maior número possível de riscos e vulnerabilidades em cadeias de suprimentos.

Por fim, encerra-se a presente revisão elencando-se a principal limitação do trabalho, ou seja, a falta de outros estudos empíricos, sobretudo no contexto nacional de pesquisa, que tenham buscado o mesmo foco de análise que norteou esta pesquisa. Especificamente, no caso da presente revisão, cabe destacar que essa falta, não só restringe as discussões sobre os modelos e suas variáveis mais importantes, 
como também inibe a possibilidade de comparação dos resultados alcançados.

\section{Referências}

ASBJORNSLEET, B. E. Assessing the vulnerability of supply chains. In: ZSIDISIN, G. A.; RITCHIE, B. (Ed.). Supply Chain Risk: A handbook of assessment, management and performance. New York: Springer, 2009.

AGUIAR, E. C. Contribuição ao estudo do fator risco no desempenho de organizações e cadeias de suprimentos. 2010. Tese (Doutorado em Administração)Faculdade de Economia, Administração e Contabilidade, Universidade de São Paulo, 2010.

BAIRD, I. S.; THOMAS, R. Toward a contingency model of strategic risk taking. Academy of Management Review, v. 10, n. 2, p. 230-243, 1985.

BLACKHURST, J. et al. An empirically derived agenda of critical research issues for managing supply chain disruptions. International Journal of Production Research, v. 43, n. 19, p. 4067-4081, 2005. http:// dx.doi.org/10.1080/00207540500151549

BLOS, M. et al. Supply chain risk management: a case study of automotive and electronic industries in Brazil. Supply Chain Management: An International Journal, v. 14, n. 4, p. 247-52, 2009. http://dx.doi. org/10.1108/13598540910970072

BRINDLEY, C. Risk focus towards customers. In: BRINDLEY, C. (Ed.). Supply Chain Risk. Hampshire: Ashgate, 2004.

BUHMAN, C.; KEKRE, S.; SINGHAL, J. Interdisciplinary and interorganizational research: Establishing the science of enterprise networks. Production \& Operations Management, v. 14, p. 493-513, 2005. http://dx.doi. org/10.1111/j.1937-5956.2005.tb00236.x

CAVINATO, J. L. Supply chain logistics risk: from de back room to the board room. International Journal of Physical Distribution \& Logistics Management, v. 34, n. 5, p. 383-389, 2004. http:// dx.doi.org/10.1108/09600030410545427

CHOPRA, S.; SODHI, M. S. Managing risk to avoid supply chain breakdown. MIT Sloan Mangement Review, v. 46, p. 53-61, n. 1, 2004.

CHRISTOPHER, M. The agile supply chain: competing in volatile markets. Industrial Market Management, v. 29 , n.1, p. 37-44, 2000. http://dx.doi.org/10.1016/ S0019-8501(99)00110-8

CHRISTOPHER, M. Logistics and supply chain management. New York: Prentice-hall, 2004.

CHRISTOPHER, M.; LEE, H. L. Supply chain confidence: the key to effective supply chains through improved visibility and reliability. Global Trade Management, p. 1-10, Nov 2001.

CHRISTOPHER, M.; LEE, H. L. Mitigating supply chain risk through improved confidence. International Journal of Physical Distribution \& Logistics Management, v. 34 n. 5, p. 388-396, 2004.

CHRISTOPHER, M.; PECK, H. Building the resilient supply chain. International Journal of Logistics Management, v. 15, n. 2, 2004. http://dx.doi. org/10.1108/09574090410700275
CHRISTOPHER, M. et al. Approaches to managing global sourcing risk. Supply Chain Management: An International Journal, v. 16, n. 2, p.67-81, 2011. http:// dx.doi.org/10.1108/13598541111115338

CRAIGHEAD, C. W. et al. 18 ways to guard against disruptions. Supply Management Review, v. 9, n. 1, p. 46-53, 2005.

CRAIGHEAD, C. W. et al. The severity of supply chain disruptions: design characteristics and mitigation capabilities. Decision Sciences, v. 38, n. 1, p. 131-156, 2007. http://dx.doi.org/10.1111/j.1540-5915.2007.00151.x

CRANFIELD SCHOOL OF MANAGEMENT. Supply chain vulnerability. Final report on behalf of DTRL, 2002.

CUCCHIELLA, F.; GASTALDI, M. Risk management in supply chain: a real option approach. Journal of Manufacturing Technology Management, v. 17, n. 6 , p. 700-720, 2006. http://dx.doi. org/10.1108/17410380610678756

DIMITURK, A. Three keys to supply chain management in times of disaster. Healthcare Purchasing News, v. 29, n. 12, p. 64-5, 2005.

DANI, S. Predicting and managing supply chain risk. In: ZSIDISIN, G. A.; RITCHIE, B. (Ed.). Supply Chain Risk: A handbook of assessment, management and performance. New York: Springer, 2009.

EASTERBY-SMITH, M.; THORPE, R.; LOWE, A. Management Research - an introduction. London: Sage Publications, 2002.

ELANGOVAN, D. et al. Development of futuristic supply chain risk management pilot strategies for achieving loss reduction in manufacturing organizations. World Journal of Entrepreneurship, Management and Sustainable Development, v. 6, n. 1-2, p. 39-51, 2011.

ELLEGAARD, C. Supply risk management in a small company perspective. Supply Chain Management: An International Journal, v. 13, n. 6, p. 425-34, 2008. http://dx.doi.org/10.1108/13598540810905688

ELKINS, D.; KULKARNI, D.; TEW, J. Identifying and assessing supply chain risk. New York: Auerbach, 2008.

FAISAL, M. N.; BANWET, D. K.; SHANKAR, R. Mapping supply chain on risk and customer sensitivity dimensions. Business Process Management Journal, v. 106, n. 6, p. 878-895, 2006.

FAISAL, M. N.; BANWET, D. K.; SHANKAR, R. Information risks management in supply chains: an assessment and mitigation framework. Journal of Enterprise Information Management, v. 20 , n. 6, p. 677-699, 2007. http://dx.doi. org/10.1108/17410390710830727

FAISAL, M. N.; BANWET, D. K.; SHANKAR, R. Supply chain risk mitigation: modeling the enablers. Business Process Management Journal, 2008.

FINCH, P. Supply chain risk management. Supply chain management: an International Journal, v. 9, n. 2, p. 183-96, 2004.

FISHER, M. et al. Configuring a supply chain to reduce the cost of demand uncertainty. Production and Operations Management, v. 6, n. 3, p. 211-225, 1997. http://dx.doi. org/10.1111/j.1937-5956.1997.tb00427.x

GHADGE, A.; DANI, S.; KALAWSKY, R. Supply chain risk management: present and future scope. 
International Journal of Logistics Management, v. 23, n. 3, p. 313-339, 2012. http://dx.doi. org/10.1108/09574091211289200

GAUDENZI, B. Assessing risks in projects and processes. In: ZSIDISIN, G. A.; RITCHIE, B Supply chain risk - a handbook of assessment, management and performance. New York: Springer, 2009

GAUDENZI, B.; BORGHESI, A. Managing risks in the supply chain using the AHP method. International Journal of Logistics Management, v. 17, n. 1, p. 114-136, 2006. http://dx.doi.org/10.1108/09574090610663464

GIUNIPERO, L. C.; ELTANTAWY, R. A. Securing the upstream supply chain: a risk management approach. International Journal of Physical Distribution \& Logistics Management, v. 34, n. 9, p. 698-713, 2004. http://dx.doi.org/10.1108/09600030410567478

HALLIKAS, J.; VIROLAINEN, V. M. Risk management in supplier relationships and network. In: BRINDLEY, C. (Ed.). Supply Chain Risk. Hampshire: Ashgate, 2004.

HALLIKAS, J.; VIROLAINEN, V. M.; TUOMINEN, M. Risk analysis and assessment in network environments: A dyadic case study. International Journal of Production Economics, v. 78, p. 45-55, 2002. http://dx.doi. org/10.1016/S0925-5273(01)00098-6

HALLIKAS, J. et al. Risk management process in supplier networks. International Journal of Production Economics, v. 90, n. 1, p. 47-58, 2004. http://dx.doi. org/10.1016/j.ijpe.2004.02.007

HALLIKAS, J.; VARIS, J. Risk management in value networks. In: ZSIDISIN, G. A.; RITCHIE, B. (Ed.). Supply Chain Risk: A handbook of assessment, management and performance. New York: Springer, 2009. PMid:19561604.

HALMAN, J. M.; KEIZER, J. A. Diagnosing risk in product innovation project. International Journal of Project Management, v. 12, n. 2, p. 75-81, 1994. http://dx.doi. org/10.1016/0263-7863(94)90013-2

HANDFIELD, R. B. A framework for reducing the impact of disruptions to the supply chain: observations for multiple executives. New York: Auerbach, 2008.

HANDFIELD, R. B.; McCORMACK, K. Consumers of supply chain risk data. New York: Auerbach, 2008.

HARLAND, C.; BRENCHLEY, R.; WALKER, H. Risk in supply networks. Journal of Purchasing \& Supply Management, v. 9, p. 51-62, 2003. http://dx.doi. org/10.1016/S1478-4092(03)00004-9

HENDRICKS, K. B.; SINGHAL, V. R. The effect of supply chain glitches on shareholder wealth. Journal of Operations Management, v. 21, p. 501-522, 2003. http://dx.doi.org/10.1016/j.jom.2003.02.003

HENDRICKS, K. B.; SINGHAL, V. R. An empirical analysis of the effect of supply chain disruption on long-run stock price performance and equity risk of the firm. Production and Operations Management, v. 14, n. 1, p. 35-52, 2005. http://dx.doi.org/10.1111/j.1937-5956.2005.tb00008.x

HANS-CHRISTIAN, P.; GALLUS, P.; THOMAS, D. Interpretive structural modeling of supply chain risks. International Journal of Physical Distribution \& Logistics Management, v. 41, n. 9, p.839-859, 2011. http://dx.doi.org/10.1108/09600031111175816
HITTLE, B.; LEONARD, K. M. Decision making in advance of a supply chain crisis. Management Decision, v. 49, n. 7, p.1182-1193, 2011. http://dx.doi. org/10.1108/00251741111151208

JIA, F.; RUTHERFORD, C. Mitigation of supply chain relational risk caused by cultural differences between China and the West. International Journal of Logistics Management, v. 21, n. 2, p. 251-270, 2010. http:// dx.doi.org/10.1108/09574091011071942

JOHNSON, M. E. Learning from toys: lessons in managing supply chain risk from the toy industry. California Management Review, v. 43 n. 3, p. 106-26, 2001. http://dx.doi.org/10.2307/41166091

JÜTTNER, U. Supply chain risk management - understanding the business requirements from a practitioner perspective. International Journal of Logistics Management, v. 16 n. 1, p. 120-41, 2005. http:// dx.doi.org/10.1108/09574090510617385

JÜTTNER, U.; CHRISTOPHER, M.; PECK, H. Supply chain risk management outlining an agenda for future research. International Journal of Logistics Management, v. 6, n. 4, p. 197-210, 2003. http://dx.doi. org/10.1080/13675560310001627016

JÜTTNER, U.; MAKLAN, S. Supply chain resilience in the global financial crisis: an empirical study. Supply Chain Management: An International Journal, v. 16, n. 4, p. 246-259, 2011. http://dx.doi. org/10.1108/13598541111139062

JÜTTNER, U.; ZIENGENBEIN, A. Supply chain risk management for small and medium- size businesses. In: ZSIDISIN, G. A.; RITCHIE, B. (Ed.). Supply Chain Risk: A handbook of assessment, management and performance. New York: Springer, 2009.

KERN, D. et al. Supply Risk Management: Model Development and Empirical Analysis. International Journal of Physical Distribution \& Logistics Management, v. 42, n. 1, 2012. http://dx.doi. org/10.1108/09600031211202472

KHAN, O.; BURNES, B. Risk and supply chain management: creating a research agenda. International Journal of Logistics Management, v. 18 n. 2, p. 197-216, 2007. http://dx.doi.org/10.1108/09574090710816931

KHAN, O.; CHRISTOPHER, M.; BURNES, B. The impact of product design on supply chain risk: a case study. International Journal of Physical Distribution \& Logistics Management, v. 38 n. 5, p. 412-32, 2008. http://dx.doi.org/10.1108/09600030810882834

KLEINDORFER, P. R. Market-based environmental audits and environmental risks. The Geneva Papers on Risk and Insurance, v. 22, n. 83, p. 194-210, 1997.

KLEINDORFER, P. R.; SAAD, G. H. Managing disruption risks in supply chains. Production \& Operations Management, v. 14, p. 53-68, 2005. http://dx.doi. org/10.1111/j.1937-5956.2005.tb00009.x

KNIGHT, F. H. Risk, uncertainty and profit. New York: Harper\&Row, 1965.

KOUVELIS, P.; CHAMBERS, C.; WANG, H. Supply chain management research and production and operations management: review, trends, and opportunities. Production \& Operations Management, v. 15, n. 3, p. 449-469, 2006. http://dx.doi.org/10.1111/j.1937-5956.2006.tb00257.x 
KOUVELIS, P. et al. Handbook of integrated risk management in global supply chains. London: Wiley, 2011. http://dx.doi.org/10.1002/9781118115800

LAEEQUDDIN, M. et al. Supply chain partners trust building process through risk evaluation: the perspectives of UAE packaged food industry. Supply Chain Management: An International Journal, v. 14, n. 4, p. 280-290, 2009. http://dx.doi.org/10.1108/13598540910970117

LAMBERT, D. M.; POHLEN, T. L. Supply chain metrics. The International Journal of Logistics Management, v. 12, n. 1, p. 1-19, 2001. http://dx.doi. org/10.1108/09574090110806190

LEE, H. L.; CHRISTOPHER, M. Mitigating supply chain risk through improve confidence. International Journal of Physical Distribution \& Logistics Management, v. 34, n. 5, p. 388-396, 2001.

LEE, H. L. A triple-A supply chain. Harvard Business Review, p. 102-112, Oct 2002.

LEVARY, R. R. Ranking foreign suppliers based on supply risk. Supply Chain Management: an International Journal, v. 12, n. 6, p. 392-394, 2007. http://dx.doi. org/10.1108/13598540710826317

LIN, Y.; ZHOU, L. The impacts of product design changes on supply chain risk: a case study. International Journal of Physical Distribution \& Logistics Management, v. 41, n. 2, p. 162-186, 2011. http:// dx.doi.org/10.1108/09600031111118549

LIU, S.; LIN, J.; HAYES, K. A. An agile and diversified supply chain: reducing operational risks. Competitiveness Review: An International Business Journal incorporating Journal of Global Competitiveness, v. 20 n. 3, p. $222-234,2010$

MANUJ, I.; MENTZER, J. T. Global supply chain risk management. Journal of Business Logistics, v. 29, n. 1, p. 133-155, 2008a. http://dx.doi. org/10.1002/j.2158-1592.2008.tb00072.x

MANUJ, I.; MENTZER, J. T. Global supply chain risk management strategies. International Journal of Physical Distribution \& Logistics Management, v. 38, p. 192-223, 2008b. http://dx.doi. org/10.1108/09600030810866986

MARBLE, R. P.; THUN, J. H. The theory of constraints and the constraints of theory: a structural equation model for supply chain disruptions. In: ANNUAL CONFERENCE OF PRODUCTION AN OPERATIONS MANAGEMENT SOCIETY, 17., 2006, Boston. Proceedings... Boston, 2006.

MASON-JONES, R.; TOWILL, D. Shrinking the supply chain uncertainty cicle. Control, p. 17-22, Sept 1998.

MATOOK, S., LASCH, R.; TAMASCHKE, R. Supplier development with benchmarking as part of a comprehensive supplier risk management framework. International Journal of Operations \& Production Management, v. 29, n. 3, p. 241-57, 2008. http://dx.doi. org/10.1108/01443570910938989

McKINNON, A. Life without Lorries: The Impact of a Temporary Disruption of Road Freight Transport in the UK, report prepared for Commercial Motors. Edinburgh: Logistics Research Centre, Heriot-Watt University, 2004.
MELNYK, S. A.; RODRIGUES, A.; RAGATZ, G. L. Using simulation to investigate supply chain disruptions. In: ZSIDISIN, G. A.; RITCHIE, B. (Ed.). Supply Chain Risk: A handbook of assessment, management and performance. New York: Springer, 2009.

MENTZER, J. T. et al. Defining supply chain management Journal of Business Logistics, v. 22, n. 2, p. 1-25, 2001. http://dx.doi.org/10.1002/j.2158-1592.2001.tb00001.x

MEULBROEK, L. The promise and challenge of integrated risk management. Risk Management and Insurance Review, v. 5, n. 1, 2003.

MITROFF, I.; ALPASAN, M. Preparing for evil. Harvard Business Review, p. 109-115, Apr 2003.

MULLAI, A. Risk management system: a conceptual model. In: ZSIDISIN, G. A.; RITCHIE, B. (Ed.). Supply Chain Risk: A handbook of assessment, management and performance. New York: Springer, 2009.

NORRMAN, A.; JANSSON, U. Ericsson's proactive supply chain risk management approach after a serious sub supplier accident. International Journal of Physical Distribution \& Logistics Management, v. 34, n. 5, p. 434-456, 2004. http://dx.doi. org/10.1108/09600030410545463

NORRMAN, A.; LINDROTH R. Categorization of Supply Chain Risk and Risk Management. In: BRINDLEY, C. Supply Chain Risk. Hampshire: Ashgate, 2004. Pmid:15644981.

OLSON, D. L.; DASH WU, D. A review of enterprise risk management in supply chain. Kybernetes, v. 39, n. 5, p. 694-706, 2010. http://dx.doi. org/10.1108/03684921011043198

OLSON, D. L.; WU, D. Risk management models for supply chain: a scenario analysis of outsourcing to China. Supply Chain Management: An International Journal, v. 16, n. 6, p.401-408, 2011. http://dx.doi. org/10.4128/9781606493311

PECK, H. Drivers of supply chain vulnerability: an integrated framework. International Journal of Physical Distribution \& Logistics Management, v. 36, n. 4, p. 210-32, 2005. http://dx.doi. org/10.1108/09600030510599904

PECK, H. Reconciling supply chain vulnerability risk and supply chain management. International Journal of Logistics: Research and Applications, v. 9, n. 2, p. 124-142, 2006. http://dx.doi. org/10.1080/13675560600673578

PERRY, M. Natural disaster management planning: a study of logistics managers responding to the tsunami. International Journal of Physical Distribution \& Logistics Management, v. 37, n. 5, p. 409-33, 2007. http://dx.doi.org/10.1108/09600030710758455

PERROW, C. Normal accidents: living with high risks technologies. Princenton University: New Jersey, 1999.

PONOMAROV, S. Y.; HOLCOMB, M. C. Understanding the concept of supply chain resilience. International Journal of Logistics Management, v. 20, n. 1, p. 124-14, 2009. http://dx.doi.org/10.1108/09574090910954873

PUJAWAN, N.; GERALDIN, L. H. House of risk: a model for proactive supply chain risk management. Business Process Management Journal, v. 15, n. 6, p. 953-967, 2009. http://dx.doi.org/10.1108/14637150911003801 
RAO, S.; GOLDSBY, T. Supply chain risks: a review and typology. The International Journal of Logistics Management, v. 20, n. 1, p. 97-123, 2009. http://dx.doi. org/10.1108/09574090910954864

RICE, B.; CAINATO, F. Building a secure and resilient supply network. Supply Chain Management Review, v. 7, n. 5, p. 22-30, 2003.

RITCHIE, B.; BRINDLEY, C. Risk characteristics of supply chain - a contingency framework. In: BRINDLEY, C. Supply chain risk. Hampshire: Ashgate, 2004.

RITCHIE, B., BRINDLEY, C. Supply chain risk management and performance: a guiding framework for future development. International Journal of Operations and Production Management, v. 27, p. 303-322, 2007. http://dx.doi.org/10.1108/01443570710725563

SANCHEZ-RODRIGUES, V.; POTTER, A.; NAIM, M. M. Evaluating the causes of uncertainty in logistics operations. International Journal of Logistics Management, v. 21, n. 1, p. 45-64, 2010. http://dx.doi. org/10.1108/09574091011042179

SHEFFI, Y. Supply chain management under threat of international terrorism. International Journal of Logistics Management, v. 12, n. 2, p. 1-11, 2001. http:// dx.doi.org/10.1108/09574090110806262

SHEFFI, Y Building a culture of flexibility. World Trade, v. 18, n. 12, p. 26-9, 2005.

SHEFFI, Y.; RICE, J.B. A supply chain view of the resilient enterprise. Sloan Management Review, v. 47, n. 1, p. 41-8, 2005.

SHIN, K. et al. Risk propagation based dynamic transportation route finding mechanism. Industrial Management \& Data Systems, v. 112, n. 1, p. 102-124, 2012. http:// dx.doi.org/10.1108/02635571211193662

SIMCHI-LEVI, D.; KAMINSKY, P.; SIMCHI-LEVI, E. Cadeia de suprimentos - Projeto e Gestão. Porto Alegre: Bookman, 2000.

SKIPPER, J. B.; HANNA, J. B. Minimizing supply chain disruption risk through enhanced flexibility. International Journal of Physical Distribution \& Logistics Management, v. 39, n. 5, p. 404-427, 2009. http://dx.doi.org/10.1108/09600030910973742

SMITH, M. E. Psychological foundations of supply chain risk management. In: ZSIDISIN, G. A.; RITCHIE, B. (Ed.). Supply Chain Risk: A handbook of assessment, management and performance. New York: Springer, 2009.

SPECKMAN, R. E.; DAVIS, E. W. Risky business: expanding the discussion on risk and the extended enterprise. International Journal of Physical Distribution \& Logistics Management, v. 34, n. 5, p. 414-33, 2004. http://dx.doi.org/10.1108/09600030410545454

SRIVASTAVA, S. Green supply chain management: a state-of-the-art literature review. International Journal of Review, v. 9, n. 1, p. 53-80, 2007. http://dx.doi. org/10.1111/j.1468-2370.2007.00202.x

SODHI, M. Managing demand risk in tactical supply chain planning for a global consumer electronics company. Production and Operations Management, v. 14, n. 1, p. 69-79, 2005. http://dx.doi. org/10.1111/j.1937-5956.2005.tb00010.x

SVENSSON, G. A conceptual framework for the analysis of vulnerability in supply chains. International
Journal of Distribution \& Logistics Management, v. 30, n. 9, p. 731-749, 2000. http://dx.doi. org/10.1108/09600030010351444

SVENSSON, G. The impact of outsourcing on inbound logistics flow. The International Journal of Logistics Management, v. 12, n. 1, p. 21-35, 2002a. http://dx.doi. org/10.1108/09574090110806208

SVENSSON, G. A conceptual framework of vulnerability in firm inbound and outbound logistics flows. International Journal of Physical Distribution \& Logistics Management, v. 32, n. 2, p. 110-34, 2002b. http:// dx.doi.org/10.1108/09600030210421723

SVENSSON, G. Vulnerability scenarios in marketing channels. Supply Chain Management: An International Journal, v. 7, n. 5, p. 322-33, 2002c. http://dx.doi. org/10.1108/13598540210447733

SVENSSON, G. A Typology of Vulnerability Scenarios towards Suppliers and Customers in Supply Chains based upon Perceived Time and Relationship Dependence. International Journal of Physical Distribution \& Logistics Management, v. 32, n. 3, p. 168-187, 2002d. http://dx.doi.org/10.1108/09600030210426511

SVENSSON, G. Vulnerability in business relationships: the gap between dependence and trust. Journal of Business and Industrial Marketing, v. 19, n. 7, p. 469-483, 2003a http://dx.doi.org/10.1108/08858620410564418

SVENSSON, G. Key areas, causes and contingency planning of corporate vulnerability in supply chains -a qualitative approach. International Journal of Physical Distribution \& Logistics Management, v. 34, n. 9, p. 728-748, 2003b. http://dx.doi. org/10.1108/09600030410567496

TANG, C. S. Robust strategies for mitigating supply chain disruptions. International Journal of Logistics: Research and Applications, v. 9, n. 1, p. 33-45, 2006 a. http://dx.doi.org/10.1080/13675560500405584

TANG, C. S. Perspectives in supply chain risk management. International Journal of Production Economics, v. 103, n. 2, p. 451-488, 2006b. http://dx.doi.org/10.1016/j. ijpe.2005.12.006

TANG, C.; TOMLIN, B. The power of flexibility for mitigating supply chain risks. International Journal of Production Economics, v. 116, p. 12-27, 2008. http:// dx.doi.org/10.1016/j.ijpe.2008.07.008

TOMLIN, B. On the value of mitigation and contingency strategies for managing supply chain disruption risks. Management Science, v. 52, n. 5, p. 639-657, 2006. http://dx.doi.org/10.1287/mnsc.1060.0515

TOWILL, D. R. The impact of business policy on bullwhip induced risk in supply chain Management. International Journal of Physical Distribution \& Logistics Management, v. 35, n. 8, p. 555-75, 2005. http://dx.doi.org/10.1108/09600030510623339

TUMMALA, R.; SCHOENHERR, T. Assessing and managing risks using the Supply Chain Risk Management Process (SCRMP). Supply Chain Management: An International Journal, v. 16, n. 6, p. 474-483, 2011. http://dx.doi.org/10.1108/13598541111171165

VISWANADHAM, N.; GAONKAR, R. S. Risk management in global supply chain networks. The 
Logistics Institute - Asia Pacific, National University of Singapore, 2008.

WAGNER, S. M.; BODE, C. An empirical investigation into supply chain vulnerability. Journal of Purchasing \& Supply Management, v. 12, n. 6, p. 301-12, 2006. http://dx.doi.org/10.1016/j.pursup.2007.01.004

WAGNER, S. M.; BODE, C. An empirical examination of supply chain performance along several dimensions of risk. Journal of Business Logistics, v. 29 , n. 1, p. 307-325, 2008. http://dx.doi. org/10.1002/j.2158-1592.2008.tb00081.x

WU, T.; BLACKHURST, J.; CHIDAMBARAM, V. A modelo for inbound supply risk analysis. Computers in Industry, v. 57, p. 350-365, 2006. http://dx.doi. org/10.1016/j.compind.2005.11.001

ZENG, A. Z.; BERGER, P. D.; GERSTENFELD, A. Managing the supply-side risks in supply chains. Berlin: Springer, 2005.

ZSIDISIN, G. A. Managerial perceptions of supply risk. Journal of Supply Chain Management, v. 39, n. 1, p. 14-25, 2003. http://dx.doi.org/10.1111/j.1745493X.2003.tb00146.X

ZSIDISIN, G. A. A grounded definition of supply risk. Journal of Purchasing and Supply Management, v. 9 n. 5-6, p. 217-224, 2004. http://dx.doi.org/10.1016/j. pursup.2003.07.002

ZSIDISIN, G. A.; ELLRAM, L. M. An agency theory investigation of supply risk management. The Journal of
Supply Chain Management, v. 39, n. 3, p. 15-27, 2003. http://dx.doi.org/10.1111/j.1745-493X.2003.tb00156.x ZSIDISIN, G. A.; SMITH, M. Managing supply risk with early supplier involvement - a case study and research propositions. The Journal of Supply Chain Management, v. 41, n. 4, p. 44-57, 2005. http://dx.doi. org/10.1111/j.1745-493X.2005.04104005.x

ZSIDISIN, G. A. et al. An Analysis of Supply Risk Assessment Techniques. International Journal of Physical Distribution and Logistics Management, v. 34, n. 5, p. 397-413, 2004. http://dx.doi. org/10.1108/09600030410545445

ZSIDISIN, G. A.; MELNYK, S. A.; RAGATZ, G.L. An institutional theory perspective of business continuity planning for purchasing and supply management. International Journal of Production Research, v. 43, n. 16, p. 3401-3420, 2005. http://dx.doi. org/10.1080/00207540500095613

ZSIDISIN, G. A.; PANELLI, A.; UPTON, R. Purchasing organization involvement in risk assessments, contingency plans, and risk management: an exploratory study. Supply Chain Management: An International Journal, v. 5 n. 4, p. 187-197, 2000. http://dx.doi. org/10.1108/13598540010347307

ZSIDISIN, G. A.; RITCHIE, B. Supply chain risk management - developments, issues and challenges. In: Supply chain risk - a handbook of assessment, management and performance. Springer: New York, 2009. 